\title{
Clinical Evidence of Herbal Drugs As Perpetrators of Pharmacokinetic Drug Interactions
}

Authors

Affiliations
Robert Hermann ${ }^{1}$, Oliver von Richter ${ }^{2}$

${ }^{1}$ Clinical Research Appliance, Radolfzell, Germany

${ }^{2}$ Merck Serono, Darmstadt, Germany

\author{
Key words \\ - herbal drugs \\ - botanical drugs \\ - complementary and \\ alternative medicines \\ - drug interactions \\ - pharmacokinetics
}

received February 28, 2012 revised June 22, 2012 accepted July 2, 2012

Bibliography

DOI http://dx.doi.org/

10.1055/s-0032-1315117

Published online August 1, 2012

Planta Med 2012; 78 :

1458-1477 @ Georg Thieme

Verlag KG Stuttgart · New York . ISSN 0032-0943

\section{Correspondence}

Dr. Robert Hermann

Clinical Research Appliance

Rossittenstraße 15

78315 Radolfzell

Germany

Phone: +497732820951

Fax: +497732820953

robert.hermann@

cr-appliance.com

\section{Abstract \\ $\checkmark$}

The use of herbal/botanical products, also referred to as complementary and alternative medicines (CAM), worldwide enjoys increasing popularity. It appears in particular highly prevalent in patient populations already exposed to complex treatment algorithms and polypharmacotherapy, frequently involving narrow therapeutic index drugs. Accordingly, the potential clinical dimension and relevance of herb-drug interactions has received considerable attention over the last years. However, review of pertinent literature indicates that the available clinical evidence in this regard is still limited and sometimes inconclusive. Also, communication of herb-drug interaction data in the biopharmaceutical/medical literature is often complex and confusing, not always unbiased, and in many cases appears not to strive for clear-cut and useful guidance in terms of the clinical relevance of such findings.

This systematic review summarizes and interprets the published evidence on clinical herbdrug interaction studies which examined the potential of six popular herbal drugs (Echinacea, garlic, gingko, ginseng, goldenseal, and milk thistle) as perpetrators of pharmacokinetic (PK) drug interactions. Reported effect sizes were systematically categorized according to FDA drug interaction guideline criteria. A total of 66 clinical PK interaction studies, meeting the scope of the present review, were identified. The clinical evidence was found to be most robust and informative for Gingko biloba (GB; 21 studies) and milk thistle/silymarin (MT; 13), and appears still limited for ginseng (9), goldenseal/berberine (GS; 8), garlic (8), and Echinacea (7). Collectively, the available evidence indicates that, at commonly recommended doses, none of these herbs act as potent or moderate inhibitors or inducers of cytochrome P450 (CYP) enzymes or P-glycoprotein (ABCB1). Weak effects in terms of either induction or inhibition were found for GB (presystemic/hepatic CYP3A4 induction/inhibition, CYP2C19 induction at high doses), milk thistle/silymarin (CYP2C9 inhibition), GS/berberine (CYP3A4 and CYP2D6 inhibition), Echinacea (presystemic/hepatic CYP3A4 inhibition/induction, CYP1A2 and CYP2C9 inhibition at high doses). Information was found not always complete for the major drug metabolizing CYP enzymes in the less wellstudied herbs and is largely limited to P-glycoprotein $(A B C B 1)$ when effects on drug transporters have been investigated.

\section{Abbreviations}

$\nabla$

1-OH-MDZ: 1'hydroxy midazolam

6-OHCZX: 6-hydroxy chlorzoxazone

ABCB1: $\quad$ ATP binding cassette transporter B1 (P-glycoprotein)

BBR: berberine

b.i.d.: twice daily

BP: botanical products

CAM: complementary and alternative medicine

CAR: constitutive androstane receptor

CL/F: apparent oral clearance

CYP: $\quad$ cytochrome P450 enzyme

CZX: chlorzoxazone

DDI: drug-drug interaction

EH: epoxide hydrolase

EM: $\quad$ extensive metabolizer

GST: glutathione S-transferase

IR: immediate release

MDZ: midazolam

NHP: $\quad$ natural health product

Nrf2: $\quad$ E2-related factor 2

OSC: $\quad$ organo sulfur compounds

PD: pharmacodynamics

P-gp: $\quad$ P-glycoprotein (ABCB1)

PK: $\quad$ pharmacokinetics

PM: $\quad$ poor metabolizer 
p.o.:

PXR:

q. d.:

per oral

pregnane $\mathrm{X}$ receptor

once daily
QR:

t.i.d.:

UGT: quinine reductase

three times daily

uridine diphosphate (UDP)-glucuronosyltransferase

\section{Introduction}

Historically, plants represent the origin of modern pharmacotherapy. Herbal or botanical drug products are complex mixtures of organic chemicals that are derived from raw or processed parts of plants, such as seeds, roots, bark, leaves, flowers or fruits, or mixtures thereof [1]. Many herbal products have been used for thousands of years, e.g., in traditional herbal medicine across Asia, Australia, South America. Likewise, herbal products enjoy increasing popularity as an essential part of the trend towards complementary and alternative medicine (CAM) practices in Europe and North America. The use of herbal drugs varies by regions and patient populations: In Western countries it was estimated to occur with a prevalence of $12.1-18.6 \%$ in the United States [2] and 9.0-23.2\% in Canada [3] among the general adult population [4]. The concomitant intake of herbal drugs has been identified to occur in $16 \%$ of prescription drug users in the United States [5], whereas in Canada the combined use of herbal and prescription drugs was reported to be lower at 5.3\% [4]. Many consumers in these countries perceive herbal drugs as natural and therefore as generally safe. However, the naturalness of herbal drugs does not guarantee that they are generally safe, free from adverse effects or toxicity, and devoid of any drug-drug interaction potential. Furthermore, herbal drugs represent a mixture of organic compounds that are cleared by the same system of xenobiotic metabolism and transport that eliminate synthetic drugs from the human body. Therefore, from a mechanistic perspective, pharmacokinetic (PK) herb-drug interactions appear inevitable and comparable with those known for drug-drug interactions with synthetic pharmaceutical products.

In today's drug development process of synthetic drugs, the assessment of drug-drug interactions comprises a systematic combination of in vitro and in vivo investigations aiming at identifying possible interactions with in vitro screens which are then followed up by dedicated in vivo studies. Thereby the role of in vitro investigations and perhaps nonclinical PK interaction studies is only to inform the clinical drug-drug interaction program, not to serve as substitutes for clinical drug-drug interaction studies. Nonclinical studies in animals are generally discouraged due to the marked species differences, which renders extrapolation of such results to humans difficult [6]. Extrapolation of in vitro data to the in vivo situation by physiologically-based pharmacokinetic modelling (PBPK), which is being used to guide in vivo drug-drug interaction trials and drug labelling, is difficult to apply to herbal drugs because it requires I) the identification of the herbal component(s) acting as a perpetrator of the herb-drug interaction, II) content uniformity of these components within and across marketed products, III) well designed in vitro studies characterizing the DDI potential, and IV) most importantly, knowledge about the systemic bioavailability and human PK of this (these) component(s). Information on PK characteristics of herbal components, however, is often lacking with only a few exceptions. Therefore, it is not surprising that the extrapolation from in vitro herb-drug interaction data, in most cases, did not correctly predict the outcome of the corresponding in vivo study, except for a few examples such as berberine (BBR), an active component of goldenseal that was shown to be a mechanism-based inhibitor of CYP3A4 in vitro [7], which actually translated into a weak inhibition of CYP3A4 activity in vivo by marketed goldenseal and BBR products in various independent studies [8-10].

Review of pertinent literature indicates that the communication of herb-drug interaction issues in the biopharmaceutical/medical literature is often confusing, hardly providing useful guidance in terms of the clinical relevance of such interactions. Therefore, the issue still represents a considerable challenge for the health care professionals and a potential safety concern for patients and/or consumers.

The aim of this article is therefore to provide a systematic review of the publicly available clinical evidence of herbal drugs as perpetrators of PK drug interactions. The review focuses on detailed information gained from mechanistic herb-drug interaction studies and aims to carefully consider trial design aspects, employed products, dosages, and treatment durations in the analysis and interpretation of the data. Thereby, it was one of the key objectives of the review to guide the reader through the interpretation of the data and to provide clear assessments of reported interactions in terms of their generalizability and clinical significance. Case reports were not considered to provide robust and reliable evidence of herb-drug interactions and were therefore excluded from this review. Pharmacodynamic herb-drug interactions including overlapping toxicities, although not less important, are beyond the scope of this work but have been reviewed elsewhere [11,12]. The present review is focused on Echinacea, garlic, gingko, ginseng, goldenseal, and milk thistle ranking among the most frequently used herbal drugs in the United States [13], Canada [4], and Europe [12]. St. John's Wort is not addressed because of its well-documented properties as a perpetrator of PK-based drug interactions.

\section{Methods \\ $\nabla$}

The University of Washington Metabolism and Drug Transporter Database (DIDB) [14], SCOPUS, as well as PubMed were used to screen for 6 herb-drug interactions identifying a total of 66 clinical interaction studies. The following numbers of studies were found for the individual herbs and fruit juices: Echinacea (7), garlic (8), gingko (21), ginseng (9), goldenseal (8), and milk thistle (13).

\section{Evaluation of Herb-Drug Interactions in Humans}

$\nabla$

\section{Ginkgo}

Ginkgo (Ginkgo biloba; family Ginkgoaceae), also known as maidenhair tree, is a 190 million years old, unique species of tree with no close living relatives [15]. Pharmaceutical G. biloba (GB) products represent leave extracts and contain flavonoid glycosides (mainly quercetin, kaempferol, isorhamnetin) and terpene lactones (ginkgolides A, B, C, J, and bilobalides). GB products rank among the most widely used herbal products in the United States. Oral standardized dry extracts of GB usually contain between $22-27 \%$ flavones glycosides, 5-7\% terpene lactones, and should not exceed more than 5 ppm of ginkgolic acids, constitu- 
ents with known allergic potency [16]. Many studies have been conducted using EGb 761, a well-defined extract of GB.

GB is believed to have nootropic properties and is mainly used as a memory and concentration enhancer and for the treatment of cognitive impairment and dementia of various origins (e.g., Alzheimer's disease). GB products are also advocated for use in peripheral arterial disease (i.e., intermittent claudication) and to improve microcirculation in disease states such as tinnitus. GB supplements are usually taken in the range of 40 to $240 \mathrm{mg}$ per day, with recommended doses of 120 to $240 \mathrm{mg}$ daily for treatment of dementia and memory impairment.

GB preparations may confer appreciable antiplatelet effects, which are apparently mediated by various ginkgolides. Accordingly, the most frequently reported interactions associated with the use of GB are referring to enhancement of the PD action of platelet inhibitors or anticoagulants. Although clinically important, these PD interactions are beyond the scope of the present review on metabolism- and transporter-based PK interactions. Various and overall inconsistent in vitro effects of GB extracts or specific constituents were demonstrated regarding their potential to alter activities of CYP enzymes. Findings suggesting either induction or inhibition of various CYP enzymes, sometimes concentration-dependently (i.e., inhibition at low concentrations, induction at high concentrations), sometimes in a substrate-dependent fashion have been described. Results have been obtained in part at very high concentrations, unlikely to be achieved at recommended doses in vivo. Collectively, the accumulated evidence from in vitro studies provides unfortunately little guidance in the reliable prediction of relevant metabolic or transporter-based GB-mediated drug interactions in vivo.

In the meantime, however, a considerable number of clinical studies have been conducted for the assessment of GB effects on various CYP isoforms, other drug metabolizing enzymes, and transporters, predominantly by using specific probe drugs, thereby allowing the identification and quantification of alterations of specific CYP enzyme and transporter activities. Overall, we identified a total of 21 human PK studies, reporting original drug interaction data on GB products.

Gurley and coworkers evaluated the effect of GB extract (containing $24 \%$ flavone glycosides and $6 \%$ terpene lactones), given at doses of $60 \mathrm{mg}$ four times daily over 28 days to 12 healthy adult subjects (6 m/6 f) [17]. In this study, CYP 3A4, 1A2, 2E1, and 2D6 activities were estimated by the use of 1-OH-MDZ/MDZ serum ratios (1-hour sample), paraxanthine/caffeine serum ratios (6hour sample), 6-OH-CZX/CZX serum ratios (2-hour sample), and debrisoquine urinary recovery ratios (8-hour collection), respectively. Comparisons of pretreatment and post-treatment ratios indicated that GB did not result in any appreciable alteration of CYP 3A4, 1A2, and 2D6 function but appeared to modestly enhance the activity of CYP2E1 by $23 \%$, although this effect was not statistically significant [17].

In a similar study in 12 elderly subjects ( $6 \mathrm{~m} / 6 \mathrm{f})$, using the same GB product, dose and treatment duration, as well as the same CYP probe drugs, no significant effects of GB in terms of modulation of CYP 3A4, 1A2, 2E1, and 2D6 activities were found, thereby not confirming the previously observed trend towards a modest CYP2E1 induction [18].

Consistent results were reported by Markowitz and colleagues, who assessed the influence of standardized GB extract on the activity of CYP2D6 and 3A4 in normal adult subjects, phenotyped as CYP2D6 extensive metabolizers. As probe substrates, dextromethorphan (CYP2D6 activity) and alprazolam (CYP3A4 activity) were coadministered orally at baseline, and following treatment with GB (120 mg b.i.d. = $240 \mathrm{mg} /$ day $)$ for 14 days. No statistically significant differences were found between baseline and post-GB treatment on dextromethorphan metabolic ratios, indicating a lack of GB effect on CYP2D6 activity. For alprazolam there was a statistically significant, albeit modest $17 \%$ decrease in total exposure (AUC). Based on these findings, the authors concluded that standardized extracts of GB at recommended doses are unlikely to significantly alter the disposition of coadministered medications primarily relying in their clearance on CYP2D6 or CYP3A4 pathways [19].

The effects of GB supplementation ( $90 \mathrm{mg} /$ day) administered for 30 days on the steady-state plasma concentrations of the CYP3A4 and CYP2D6 substrate donepezil were investigated in 14 Japanese patients ( $6 \mathrm{~m} / 8 \mathrm{f}$; age range $65-80$ years) with Alzheimer's disease [20]. The results of this study showed that relatively low doses of GB in elderly patients with Alzheimer's disease did not alter the steady-state plasma concentrations of donepezil, which implies that daily doses of $90 \mathrm{mg}$ of ginkgo do not have appreciable inhibitory or inducing effects on the CYP3A4- and CYP2D6mediated metabolism of donepezil in the target population.

Robertson and colleagues evaluated the effect of GB (120 mg b.i.d. $=240 \mathrm{mg} /$ day) for two weeks on the steady-state exposure of lopinavir and ritonavir in 14 healthy subjects administered a lopinavir/ritonavir combination [21]. In addition, single oral doses of probe drugs MDZ and fexofenadine were administered prior to and after 4 weeks of GB (following washout of lopinavir/ ritonavir) to assess the influence of ginkgo on CYP3A4 and P-gp activity, respectively. Lopinavir, ritonavir, and fexofenadine exposures were not significantly affected by GB administration, while total (AUC) and maximum $\left(\mathrm{C}_{\max }\right)$ exposure of the CYP3A4 prototypic substrate MDZ were significantly lowered by $34 \%$ and $31 \%$, respectively, relative to baseline. These results suggest that GB modestly induces - most probably presystemic (i.e., in the gut wall) - CYP3A metabolism, as indicated by the observed decrease in MDZ bioavailability. However, there was no change in the exposure of lopinavir, suggesting that the modest GB-mediated induction of CYP3A4 has most likely been superseded by ritonavir's potent inhibition of CYP3A4. Thus, GB appears unlikely to reduce the exposure of ritonavir-boosted protease inhibitors. As the $\mathrm{H}_{1}$ receptor antagonist fexofenadine is a substrate of P-gp (ABCB1), the intestinal uptake transporters organic anion transporting polypeptides 1A2 (OATP1A2) and 2B1 (OATP2B1) as well as the hepatic uptake transporter OATP1B1 [22], the observed unaltered disposition of fexofenadine in the study suggests that GB is unlikely to alter the function of these transporter proteins to a significant extent in vivo.

In a small single-dose study in 8 healthy male subjects, $240 \mathrm{mg}$ of GB (Ginkgolon-24 $4^{\mathrm{TM}}$, powder capsules) did not alter the mean exposure to the CYP3A4 substrate nifedipine or its major metabolite dehydronifedipine [23]. Although the observation of remarkably increased nifedipine $\mathrm{C}_{\text {max }}$ levels in two subjects upon GB coadministration was emphasized by the authors, and possible underlying mechanisms discussed, the known PK variability of nifedipine together with the overall study results indicating absence of any GB effects in terms of total nifedipine exposure (i.e., AUC) rather support the assumption of a lack of a systematic effect of $G$. biloba on the bioavailability of nifedipine. This interpretation would be consistent with reports from other GB interaction studies with CYP3A4 substrates (see above).

Uchida et al. assessed the influence of high repeat doses of GB $\left(360 \mathrm{mg} / \mathrm{d}\right.$ EGb 761, Ginkgold ${ }^{\circledR}$, given as two $60 \mathrm{mg}$ tablets 
(120 mg) t.i.d. for 28 days $=360 \mathrm{mg} /$ day $)$ on the PK of orally administered CYP2C9 and CYP3A4 probe drugs tolbutamide (125 mg, single dose) and midazolam ( $8 \mathrm{mg}$, single dose) in 10 healthy male subjects, thereof 9 subjects with extensive metabolizer genotypes of CYP2C9 (CYP2C9*1/*1) and 1 subject with CYP2C9*1/*3 genotype [24]. This study showed that high-dose $\mathrm{GB}$ treatment slightly decreased the total exposure $\left(\mathrm{AUC}_{0-\infty}\right)$ of tolbutamide by $16 \%$ and the metabolic $\mathrm{AUC}_{0-\infty}$ ratio of tolbutamide to 4-hydroxytolbutamide in plasma by $17 \%$ ( $p<0.05$ for both outcomes). In contrast, total exposure $\left(\mathrm{AUC}_{0-\infty}\right.$ ) of $\mathrm{MDZ}$ was significantly increased by $25 \%$ and oral clearance significantly decreased by $26 \%$ upon repeated high-dose GB treatment, whereby the metabolic $\mathrm{AUC}_{0-\infty}$ ratio of $\mathrm{MDZ}$ to $1-\mathrm{OH}-\mathrm{MDZ}$ was not significantly altered. The study suggests evidence that a minor CYP2C9 induction can be achieved with supra-therapeutic doses (360 mg/day) of GB in vivo, and that some inhibition of intestinal CYP3A4 may go along with a modest increase in the bioavailability of sensitive CYP3A4 substrates. Overall the study results indicate a low potential of GB to confer CYP3A4- and CYP2C9-based metabolic drug interactions, and thus are consistent with reports from other studies that reported no effects of $\mathrm{GB}$ on the function of these enzymes at recommended doses.

By using diazepam as a model substrate of CYP2C19 and 3A4, when $10 \mathrm{mg}$ single diazepam doses were given either alone or concomitantly with oral GB ( $120 \mathrm{mg}$ b.i.d. $=240 \mathrm{mg} /$ day $)$ for 28 days to 12 healthy subjects, Zuo and coworkers demonstrated that total exposure (i.e., AUC) for diazepam and the main metabolite N-desmethyldiazepam were essentially unaltered, thereby indicating that the disposition of CYP2C19 and 3A4 substrates is unlikely to be substantially modified by recommended doses of GB products [25].

Jiang and colleagues investigated the effects of GB on the PK and PD of warfarin in 12 healthy male subjects (eight Caucasians, four Asians) [26]. A single 25-mg dose of warfarin (Coumadin ${ }^{\mathrm{TM}}, 5 \times 5$ mg tablets) was administered to each subject with and without pretreatment with multiple doses of GB for 1 week (Tavonin ${ }^{\mathrm{TM}}$; $40 \mathrm{mg}$ tablets, each tablet containing the standardized dry extract, EGb761, equivalent to $2 \mathrm{~g}$ of $\mathrm{G}$. biloba leaf, $9.6 \mathrm{mg}$ of ginkgo flavonglycosides, $2.4 \mathrm{mg}$ of ginkgolides and bilobalide, $2 \times 40 \mathrm{mg}$ tablets, t.i.d. $=240 \mathrm{mg} /$ day). Dosing of GB was continued for a further week after warfarin administration. The bioanalytics comprised enantiomer-selective quantification of S-warfarin, which is predominantly metabolized to S-7-hydroxywarfarin by CYP2C9, and R-warfarin, which is metabolized by CYP1A2 and CYP3A4, thereby allowing for a separate mechanistic assessment of any potential alteration of these metabolic pathways by concomitant GB treatment. The study demonstrated that GB, at the highest recommended dose, had no effect on the activity of CYP1A2, CYP3A4, or CYP2C9 in healthy subjects, as treatment did not affect the PK and clearance of both warfarin enantiomers in human subjects. GB also did not affect the apparent volumes of distribution or protein binding of warfarin enantiomers. PD endpoints of warfarin $25 \mathrm{mg}$ single doses were also assessed in this study, and it was shown that GB did not significantly alter blood coagulation (i.e., INR) outcomes and platelet aggregation.

Greenblatt and colleagues used single $100 \mathrm{mg}$ doses of the nonsteroidal anti-inflammatory drug (NSAID) flurbiprofen as a probe substrate for CYP2C9, to examine the effects of short-term GB treatment ( 3 doses of $2 \times 60 \mathrm{mg}$ tablets $=120 \mathrm{mg}$, given in 12 hour intervals, as EGb761) in 12 healthy adult subjects $(8 \mathrm{~m} / 4 \mathrm{f})$, who were taking no other medication [27]. The study showed that pretreatment of healthy subjects with usual clinical doses of GB has no detectable effect on the PK of a single dose of flurbiprofen or on the apparent extent of formation of the principal hydroxylated metabolite. The findings suggest that short-term exposure to GB does not inhibit CYP2C9 activity in vivo. As the study involved only short-term exposure to GB, it was not designed to capture possible CYP2C9 induction that could occur with long-term treatment.

Two PK studies in healthy subjects were performed using tolbutamide and diclofenac as probe substrates of CYP2C9. No interactions between GB extract and these CYP2C9 probe substrates were observed in vivo as evidenced by the lack of effect on the steady-state pharmacokinetics of diclofenac and the urinary metabolic ratio of tolbutamide [28].

Yin et al. examined the effects of GB on CYP2C19 and CYP3A4 metabolic pathways by using the proton pump inhibitor omeprazole (OPZ), as a well-established CYP2C19 substrate which undergoes CYP2C19-mediated hydroxylation to form its major metabolite 5-hydroxyomeprazole (5-OH-OPZ), and which also undergoes sulfoxidation via CYP3A4 to form omeprazole sulfone (OPZ-SUL) [29]. To decipher differential effects on both pathways, urinary $6 \beta$-hydroxycortisol/cortisol ratios were determined as a marker of CYP3A4 activity before and after GB as well. The study was conducted in 18 healthy Chinese subjects genotyped for CYP2C19 (6 homozygous EMs, 5 heterozygous EMs, and 7 homozygous PMs), and all subjects received a single oral OPZ $40 \mathrm{mg}$ dose at baseline and after a 12-day treatment period with GB ( $140 \mathrm{mg}$, two $70 \mathrm{mg}$ tablets b.i.d. $=280 \mathrm{mg} /$ day). Results displayed mean decreases in $\mathrm{OPZ} \mathrm{AUC}_{0-\infty}$ of $41.5 \%, 27.2 \%$, and $40.4 \%$ ( $p<0.05$ or 0.01 , each compared to control) in the homozygous EMs, heterozygous EMs and PMs, respectively. Similarly, decreases in OPZ-SUL $A C_{0-\infty}$ of $41.2 \%, 36.0 \%$, and $36.0 \%$ ( $p<0.05$ or 0.01 , each compared to control), respectively, were noted. However, no significant differences in the $\mathrm{AUC}_{\mathrm{OPZ}} / \mathrm{AUC}_{\mathrm{OPZ}-\mathrm{SUL}}$ metabolic ratio and the mean $6 \beta$-hydroxycortisol/cortisol ratio were observed before and after GB in each genotype group. In contrast, GB increased the plasma concentrations of 5-OH-OPZ, with mean increases of $37.5 \%, 100.7 \%$, and $232.4 \%$ in the homozygous EMs, heterozygous EMs and PMs, respectively. Accordingly, a significant decrease in the AUC ratio of OPZ to 5-OH-OPZ was observed in the homozygous EMs, heterozygous EMs, and PMs, respectively, whereby the decrease of the metabolic ratio was greater in PMs than EMs. The study results suggest that high-dose GB may induce OPZ hydroxylation in a CYP2C19 genotype-dependent manner but also appears to concurrently reduce the renal clearance of 5-OH-OPZ. Taken together, the observation that in PMs the seemingly inducing effect of GB was particularly pronounced is difficult to interpret, because of the obvious difficulty to induce CYP2C19 at all in PMs. On the other hand, the CYP3A4 activity was unaffected as shown by the lack of changes in the $\mathrm{AUC}_{\mathrm{OPZ}}$ $\mathrm{AUC}_{\mathrm{OPZ}-\mathrm{SUL}}$ as well as $6 \beta$-hydroxycortisol/cortisol ratios before and after GB. Overall, the study indicates that high-dose treatment with GB may significantly induce the clearance of CYP2C19 substrates, thereby possibly reducing their clinical effects.

Lei et al. examined the effects of GB (120 mg b.i.d. for 12 days) as an inducer of CYP2C19 in 14 healthy Chinese subjects genotyped as either CYP2C19 extensive (EMs; 7 carriers of 2C19*1/2C19*1) or poor metabolizers (PMs; 7 carriers of $2 \mathrm{C} 19^{*} 2 / 2 \mathrm{C} 19^{*} 2$ ), by assessing the single dose PK of the orally administered CYP2C19 substrate voriconazole (200 mg) [30]. In EMs, the median voriconazole $\mathrm{AUC}_{0-\infty}$ values were $5.17 \mu \mathrm{g} \times \mathrm{h} / \mathrm{mL}$ and $4.28 \mu \mathrm{g} \times \mathrm{h} / \mathrm{mL}$ after administration of voriconazole alone and after voriconazole with concomitant GB treatment, respectively, a 17\% difference 
suggesting a trend towards a modest induction of CYP2C19 by GB, although results did not achieve statistical significance. All other PK parameters of voriconazole such as $\mathrm{AUC}_{0-24}$, time to reach maximum concentration $\left(t_{\max }\right)$, terminal disposition halflife $\left(t_{1 / 2}\right)$, and apparent oral clearance $(\mathrm{CL} / \mathrm{F})$ also did not change significantly in the presence of GB in EMs of CYP2C19, and the results followed a similar pattern in PMs of CYP2C19. Based on the results of this study, it can be concluded that - even at the highest recommended doses - GB-mediated CYP2C19-based PK drug interactions appear to be weak and may have limited clinical significance.

Recently, the effects of GB (240 mg per day, administered as two 60 -mg capsules b.i.d. for 14 days) on the activity of CYP2B6 was investigated in 14 healthy adult male Chinese subjects (age range 19-25 years), by assessment of the single dose PK of the antidepressant and smoking cessation drug bupropion (150 mg), which is almost exclusively metabolized by CYP2B6 to its active metabolite hydroxybupropion. The study showed that a 14-day oral administration of GB extract at the high end of the recommended dose range had no statistically significant effect on the total exposure of bupropion or its active metabolite hydroxybupropion, as measured by plasma AUC [31]. These results indicate that GB is unlikely to alter the disposition of sensitive CYP2B6 substrates.

Mauro and coworkers assessed the effect of recommended GB doses $(240 \mathrm{mg} /$ day, administered as two $40-\mathrm{mg}$ tablets t.i.d. for 8 days) on the pharmacokinetics of the P-gp (ABCB1) substrate digoxin $(0.5 \mathrm{mg})$ in eight healthy human subjects $(7 \mathrm{~m} / 1 \mathrm{f}$, mean age $23 \pm 3$ yrs) [32]. The total digoxin exposure $\left(\mathrm{AUC}_{0-\infty}\right)$ was observed with $21.0 \pm 8.6 \mathrm{ng} / \mathrm{mL} \times \mathrm{h}$ (digoxin alone) and $25.6 \pm$ $13.2 \mathrm{ng} / \mathrm{mL} \times \mathrm{h}$ (digoxin $+\mathrm{GB})$, indicating a trend towards a modest (i.e., $22 \%$ ) increase in digoxin exposure associated with a 1week GB pretreatment. Although the authors emphasized that the digoxin AUC outcomes were not significantly different from each other, absence of GB treatment on P-gp (ABCB1) function should not be concluded from the study due to the lack of statistical power (small sample size).

The capacity of high-dose GB treatment (360 mg/day for 14 days) to alter the function of P-gp (ABCB1) was examined by Fan and coworkers in 10 healthy male subjects by using the P-gp (ABCB1) substrate talinolol (100 mg, single dose) [33]. GB increased the maximum observed plasma concentration of talinolol $\left(\mathrm{C}_{\max }\right)$ by $36 \%$ (90\% CI 10 to 68; p=0.025), $\mathrm{AUC}_{0-24}$ by $26 \%$ (90\% CI 11 to $43 ; \mathrm{p}=0.008)$, and $\mathrm{AUC}_{0-\infty}$ by $22 \%(90 \% \mathrm{CI} 8$ to $37 ; \mathrm{p}=0.014)$, respectively, without significant changes in terminal disposition half-life $\left(t_{1 / 2}\right)$ and the time to $C_{\max }\left(t_{\max }\right)$. The authors concluded that the results indicate that repeated supra-therapeutic doses of GB may modestly inhibit intestinal P-gp, thereby increasing the bioavailability of sensitive P-gp transporter substrates. Comparable results were obtained by the same group of investigators in another study of similar design [34].

The long-term administration of GB (EGb $761120 \mathrm{mg} /$ day for 90 days) to 10 healthy adult subjects and 10 patients with type 2 diabetes mellitus (T2DM) did not significantly affect the PK of metformin single doses (500 mg in healthy subjects and 250 to $850 \mathrm{mg}$ in T2DM patients) [35]. As metformin is a substrate of the plasma membrane monoamine transporters (PMAT), which are localized on the luminal side of enterocytes and were recently suggested to be the major transporter system responsible for the uptake of metformin from the gastrointestinal tract [36], the study suggests that long-term treatment with intermediate GB doses does not alter PMAT-mediated metformin absorption. As metformin, which is a hydrophilic organic cation (pKa 12.4) that is not significantly metabolized in humans is a good probe drug for organic cation transport (e.g., OCT1 and OCT3) in intestinal drug absorption, hepatic uptake (OCT1), and is also a substrate for several other polyspecific tubular OCT transporters in the kidney (e.g., OCTN1, OCTN2, MATE1, and MATE2-K may be involved in the renal excretion of metformin in humans) [37], it can be concluded that GB, at the dose level investigated, is unlikely to alter the disposition of OCT substrates to a significant extent.

Eight healthy subjects $(4 \mathrm{~m} / 4 \mathrm{f})$ were treated with single oral $250 \mathrm{mg}$ doses of ticlopidine, a putative substrate of the organic anion transporting polypeptide OATP-B, either given alone or together with repeat dose GB (40 mg t.i.d.=120/day; unspecified product) for 3 days [38]. In this study, short-term and low-dose coadministration of GB did not significantly change any of ticlopidine's PK parameters. These results are consistent with another study in which single coadministration of GB extract $(80 \mathrm{mg}$ ) with ticlopidine $(250 \mathrm{mg}$ ) in healthy male Korean subjects was not associated with any significant changes in the PK profile of ticlopidine compared with ticlopidine administered alone [39]. The information that can be derived from both studies in terms of clinical significance is limited, because of the low GB doses employed, and the short-term/single dose posology, respectively.

In the most recent study, a cocktail phenotyping design was employed to assess the interaction profile of GB extract EGb $761{ }^{\circledR}$ on the activities of the major CYP enzymes CYP1A2, CYP2C9, CYP2C19, CYP2D6, and CYP3A [40]. EGb $761{ }^{\circledR}$ extract or matched placebo were administered for 8 days to 18 healthy subjects $(8 \mathrm{~m} /$ $10 \mathrm{f}$ ) according to a 3-fold crossover design; total daily doses of $240 \mathrm{mg}$ EGb $761^{\circledR}$ were given in one study period following a $120 \mathrm{mg}$ b.i.d. posology (=240 mg/day), and in the other study period $240 \mathrm{mg}$ doses given q.d. in the morning. The phenotyping cocktail was orally administered before and after the EGb $761^{\circledR} /$ placebo treatment periods and employed the following probe substances and metrics: $150 \mathrm{mg}$ caffeine (CYP1A2, paraxanthine/caffeine plasma ratio 6 -h postdose), $125 \mathrm{mg}$ tolbutamide (CYP2C9, plasma concentration 24-h postdose), $20 \mathrm{mg} \mathrm{OPZ}$ (CYP2C19, OPZ/5-OH-OPZ plasma ratio 3-h postdose), $30 \mathrm{mg}$ dextromethorphan (CYP2D6, dextromethorphan/dextrorphan plasma ratio 3-h postdose), and $2 \mathrm{mg}$ of MDZ (CYP3A, MDZ plasma concentration 6 -h postdose).

Point estimates of EGb $761^{\circledR} /$ placebo ratios for phenotyping metrics were close to unity for all CYPs, except for CYP2C9 (0.834 for $120 \mathrm{mg}$ b.i.d.; 0.848 for $240 \mathrm{mg}$ q.d.) and 2C19 (0.874 for $120 \mathrm{mg}$ b.i.d. and $0.896240 \mathrm{mg}$ q.d.), which may suggest a weak trend towards induction of these CYP2C family enzymes. Respective CIs for most CYP enzymes were within the prespecified acceptance margins (i.e., $0.70-1.43$ ) for all ratios except CYP2C19 for EGb $761{ }^{\circledR} 120 \mathrm{mg}$ b.i.d. (90\% CI 0.681-1.122) and CYP2D6 for EGb $761{ }^{\circledR} 240 \mathrm{mg}$ q.d. (90\% CI 0.667-1.281). Taken together the data clearly indicate the absence of any CYP inhibitory potential of $240 \mathrm{mg}$ daily doses of EGb $761^{\circledR}$ extract towards CYP1A2, CYP2C9, CYP2C19, CYP2D6, and CYP3A, regardless whether the product is given once or twice daily. The study also showed that EGb $761^{\circledR}$ extract does not induce the activity of CYP1A2, CYP2D6, and CYP3A enzymes, and may confer a weak induction of CYP2C9 and CYP2C19 enzymes, which, however, appears too small to be of clinical relevance.

Taken together, besides St. John's Wort, GB appears to be at the time being, the second best studied natural health product, in terms of the clinical investigation of its metabolic and transporter-based PK drug interaction potential. The available set of published mechanistic drug-drug interaction studies allows rea- 
sonable inferences on the respective drug metabolizing enzyme and transporter effects of GB products in vivo. The information which can be derived from these studies is largely consistent, with some minor discrepancies that may be explained by differences in study design and populations, employed doses and treatment durations, and perhaps also differences in the composition of products investigated.

Collectively, the available evidence indicates that recommended doses of GB up to daily doses of $240 \mathrm{mg}$ do not have significant or clinically meaningful effects on the activity of CYP enzymes such as CYP1A2, CYP2C9, CYP2D6, CYP2B6, CYP2E1, and CYP3A4. While data on PK alterations of CYP3A4 are not entirely consistent, sometimes suggesting the possibility of presystemic induction of CYP3A4 with a high-dose treatment of GB, sometimes - in turn - suggesting CYP3A4 inhibition, the overall CYP3A4-related effect sizes described are generally weak and unlikely to be of clinical relevance unless CYP3A4 substrates with a narrow therapeutic index are concerned.

CYP2C19 appears to be modestly inducible by GB products, with across study evidence that this effect appears to emerge at the highest recommended dose level of $240 \mathrm{mg} /$ day [30] and may become somewhat more pronounced with increasing doses [29]. Differences in the effect sizes of CYP2C19 induction, however, may also depend on the individual CYP2C19 genotype of subjects [29] and perhaps on substrate characteristics. The available evidence in this respect, however, appears to be not entirely consistent and requires further investigation.

Although not as extensively studied as the metabolic interactions, the overall propensity of recommended doses of GB products to alter the functionality of transporter proteins appears low. Therapeutic and supra-therapeutic G. biloba doses (i.e., 240 and $360 \mathrm{mg} /$ day) were shown to modestly increase the bioavailability of the P-glycoprotein (ABCB1) probe substrates digoxin and talinolol by about $22 \%$ to $26 \%[33,34]$. A study employing G. biloba treatment with $240 \mathrm{mg} /$ day, however, did not show any alterations in the disposition of fexofenadine, which is a substrate of P-glycoprotein (ABCB1), the intestinal uptake transporters organic anion transporting polypeptide 1A2 (OATP1A2) and 2B1 (OATP2B1), and the hepatic uptake transporters OATP1B1 [41], thereby indicating that GB is unlikely to alter the function of these transporter proteins to a significant extent in vivo [21]. Similarly intermediate doses of GB (120 mg/day) do not appear to alter the function of membrane monoamine transporters (PMAT), organic cation transporters (e.g., OCT1 and OCT3), and other polyspecific tubular OCT-transporters in the kidney (e.g., OCTN1, OCTN2, MATE1, and MATE2-K), as shown by a recent metformin interaction study [37].

\section{Milk thistle}

Silybum marianum (family Asteraceae/Compositae), commonly known as milk thistle (MT), is one of the oldest and most extensively studied plants in the treatment of alcoholic, toxic, and viral liver diseases. Other treatment claims include prevention/amelioration of drug-induced liver toxicity (DILI), e.g., due to cancer chemotherapy, nonalcoholic fatty liver disease (NAFL), or steato hepatitis (NASH), lowering of cholesterol levels, and improvement of insulin resistance in patients with type 2 diabetes (T2DM) [42]. The active extract of MT is denoted as silymarin and represents a mixture of the flavonolignan constituents sylibin (silibinin), isosilybin, silidianin, and silichristin. Silymarin is extracted from dried MT seeds, in which it is contained at higher concentrations than in other parts of the plant. Silymarin has structural similarities to steroid hormones, which may be linked to its pharmacological actions. Silybin is the predominant and pharmacologically most active component, constituting approximately $60 \%$ to $70 \%$ of the isomers, followed by silychristin $(20 \%)$, and silydianin (10\%) [43]. The silymarin content in MT extracts may range from $40 \%$ to $80 \%$ [43]. Customary daily doses of MT extract range between 210 to $800 \mathrm{mg}$, depending on patient characteristics and therapeutic objectives.

In vitro studies implicate that silymarin and silybin can display inhibition of a variety of CYP enzymes such as CYP3A4, CYP2C8, CYP2C9, CYP2C19, CYP2D6, or CYP2E1, and also a couple of UGTs, such as UGT1A1, 1A6, 1A9, 2B7, and 2B15 [44-47]. In contrast, there is no conclusive in vitro evidence that MT would have significant effects on the activity of P-gp (ABCB1) [45, 48].

The available evidence from in vitro studies, has been acknowledged by clinical researches, and a number of clinical studies have been conducted for the in vivo assessment of the effects of MT products on various CYP isoforms, other drug metabolizing enzymes, and transporters, predominantly by using specific probe drugs, thereby allowing the identification and characterization of MT effects on specific metabolic and disposition pathways. Altogether, we identified a total of 13 pharmacokinetic drug interactions with orally administered MT extract in the public domain.

In the oldest published human MT interaction study, administration of silymarin at the lower end of usually recommended daily doses (Legalon ${ }^{\circledR}, 70 \mathrm{mg}$ t.i.d. $=210 \mathrm{mg} /$ day) for 28 days to 16 healthy subjects had no effect on the PK of aminopyrine or phenylbutazone, two nonspecific CYP probes [49].

Gurley and colleagues studied 12 healthy subjects ( $6 \mathrm{~m} / 6 \mathrm{f})$, who were randomly assigned to receive MT (175 mg b.i.d. $=350 \mathrm{mg} /$ day, standardized to $80 \%$ silymarins) for 28 days [50]. Probe drug cocktails of midazolam (MDZ; CYP3A4) and caffeine (CYP1A2), followed 24 hours later by chlorzoxazone (CYP2E1) and debrisoquine (CYP2D6), were administered before (baseline) and at the end of supplementation. Presupplementation and postsupplementation assessments of CYP3A4, CYP1A2, CYP2E1, and CYP2D6 activities were made by using 1-hydroxymidazolam (1$\mathrm{OH}-\mathrm{MDZ}$ )/MDZ serum ratios (1-hour sample), paraxanthine/caffeine serum ratios (6-hour sample), 6-hydroxychlorzoxazone/ chlorzoxazone serum ratios (2-hour sample), and debrisoquine urinary recovery ratios (8-hour collection), respectively. The results of this study indicate that daily doses of $350 \mathrm{mg}$ of a standardized MT extract did not alter human CYP1A2, CYP2D6, CYP2E1, and CYP3A4 enzyme activities in vivo and thus, do not appear to pose a significant interaction potential for substrates of these enzymes [50].

In another study conducted by the same group of investigators, 19 healthy young subjects ( $10 \mathrm{~m} / 9 \mathrm{f}$ ) received substantially higher daily doses of a standardized MT supplement for 14 days (900 mg/day). Subjects also received rifampin (300 mg b.i.d.) and clarithromycin (500 mg b.i.d.) for 7 days as positive controls for CYP3A induction and inhibition, respectively. Oral MDZ ( $8 \mathrm{mg}$ ) was administered as a CYP3A4 probe before and after supplementation and control periods [51].

In contrast to the positive controls rifampin and clarithromycin, no significant changes in MDZ PK were observed as a result of MT supplementation. Likewise, comparisons of 1-hour 1$\mathrm{OH}-\mathrm{MDZ} / \mathrm{MDZ}$ ratios before and after supplementation/medication mirrored the effects observed for MDZ concentration-time profiles, i.e., no significant changes in mean 1-hour 1-OH-MDZ/ MDZ serum ratios were noted after a 14-day course of MT 
(900 g/day), thereby confirming that MT extract is not a clinically relevant modulator of CYP3A4 activity in vivo.

Likewise, coadministration of $560 \mathrm{mg}$ silymarin (4 silymarin $140 \mathrm{mg}$ capsules, Legalon ${ }^{\circledR}$ ) administered 10 hours and 1.5 hours prior to the administration of the CYP3A4 substrate nifedipine (10 mg IR tablet) to 16 young healthy male subjects did not considerably change the extent of absorption or metabolism of nifedipine. Overall, the total extent of nifedipine exposure (i.e., AUC) was $13 \%$ higher $(90 \% \mathrm{CI} 0.97$ - to 1.32$)$ in the silymarin period, while $C_{\max }$ values were on average $30 \%$ lower $(90 \% \mathrm{CI} 0.39$ - to 1.27), consistent with a trend towards a delayed nifedipine absorption in the silymarin period [52]. On the whole, also this study confirmed that silymarin does not act as a potent CYP3A4 inhibitor in vivo. The data suggest modest effects of silymarin on the absorption characteristics of nifedipine, resembling the known pattern of nifedipine food effects. The authors discussed that underlying mechanisms could be either a delay in the gastric emptying rate by silymarin or inhibition of OATP transporters expressed in the GI tract [52].

The effects of a 14-day treatment with a 900-mg daily dose of a standardized MT extract ( $300 \mathrm{mg}$ t. i.d., standardized to contain $80 \%$ silymarin per capsule) on the activity of CYP2D6 were investigated in 16 healthy subjects $(8 \mathrm{~m} / 8 \mathrm{f})$, by administration of the CYP2D6 substrate debrisoquine $(5 \mathrm{mg})$ before and at the end of MT supplementation, by using 8-h debrisoquine urinary recovery ratios [53]. Results did not indicate significant inhibition of CYP2D6 by MT supplementation (post/pre geom. mean ratio $0.97,90 \%$ CI 0.93 to 1.10 ).

Two PK interaction studies, each of them conducted in 10 healthy male and female subjects, examined if MT extract may have the potential to alter the PK of the HIV protease inhibitor and CYP3A4 and P-gp substrate indinavir $[54,55]$.

In the first study, subjects received four oral doses of indinavir $800 \mathrm{mg}$ every 8 hours for baseline PK, followed by a 3-week MT treatment $(175 \mathrm{mg}$ tablets t.i. d. $=525 \mathrm{mg} /$ day; each tablet containing $153 \mathrm{mg}$ of silymarins, equivalent to $191 \mathrm{mg}$ of MT extract standardized for $80 \%$ silymarins; Thisilyn). Then the course of four oral doses of indinavir $800 \mathrm{mg}$ every 8 hours was repeated on top of continued MT treatment, and results were compared with baseline indinavir PK outcomes [55]. Indinavir plasma concentrations were followed for $8 \mathrm{~h}$ postdose. Results showed that regular MT treatment over 3 weeks did not significantly alter the overall exposure to indinavir, as evidenced by a nonsignificant average $9 \%$ reduction in indinavir $+\mathrm{MT} \mathrm{AUC}_{0-8}$ compared to indinavir alone treatment at baseline. On examination of individual patients, the $\mathrm{AUC}_{0-8}$ of indinavir in the presence of MT was $70-107 \%$ of the $\mathrm{AUC}_{0-8}$ for indinavir alone. Average indinavir trough values taken at 8 hours postdose, displayed a statistical significant, albeit modest average decline of about $25 \%$ upon MT treatment.

In the second indinavir/MT interaction study, indinavir $800 \mathrm{mg}$ t.i.d. was given for four doses, followed by MT $160 \mathrm{mg}$ t.i.d. $(=480 \mathrm{mg} /$ day standardized MT) for 13 days, with both drugs given at the same dosages over the following 2 days [54]. Indinavir plasma concentrations were followed up to 5 hours postdose. When given alone and combined with MT, respectively, the geometric mean $(95 \%$ CIs) steady-state indinavir AUC was $20.7 \mathrm{hr}$. $\mathrm{mg} / \mathrm{L}(15.3-28.2 \mathrm{hr} \cdot \mathrm{mg} / \mathrm{L})$ and $19.4 \mathrm{hr} \cdot \mathrm{mg} / \mathrm{L}(15.8-23.6 \mathrm{hr} \cdot \mathrm{mg} /$ $\mathrm{L}$ ), and the trough plasma concentrations were $0.340 \mathrm{mg} / \mathrm{L}$ $(0.232-0.497 \mathrm{mg} / \mathrm{L})$ and $0.232 \mathrm{mg} / \mathrm{L}(0.129-0.419 \mathrm{mg} / \mathrm{L})$. Average observed maximum exposure $\left(C_{\max }\right)$ was $8.85(7.17-10.9) \mathrm{mg} / \mathrm{L}$ and $7.85 \mathrm{mg} / \mathrm{L}(6.55-9.30)$ for indinavir alone and indinavir + si- lymarin, respectively. None of the PK outcomes for indinavir indicated a statistical significant alteration by MT treatment, and the authors concluded that a daily dosage of $480 \mathrm{mg}$ of a standardized silymarin extract has no apparent effect on indinavir plasma concentrations. Taken together, both indinavir interaction studies yielded consistent results, thereby indicating that MT supplementation at commonly recommended doses does not alter the activity of CYP3A4 in vivo to a clinically relevant extent.

A study with another CYP3A4 and putative P-gp substrate, the $\mathrm{H}_{2}$ receptor antagonist ranitidine, also yielded a consistent outcome in that regard [56]. In this trial, 12 healthy male subjects received oral ranitidine $150 \mathrm{mg}$ either alone or after a 7-day pretreatment with silymarin $140 \mathrm{mg}$ t.i.d. ( $=420 \mathrm{mg} /$ day). There was no influence of silymarin coadministration on the pharmacokinetics of ranitidine (i.e., $\mathrm{C}_{\max }$ and $\mathrm{AUC}$ ).

In a sample of 6 cancer patients, van Erp and colleagues investigated whether short-term MT supplementation affects the PK of the anticancer drug irinotecan, a substrate for CYP3A4 and UGT1A1 enzymes [57]. Patients were treated with irinotecan (dose $125 \mathrm{mg} / \mathrm{m}^{2}$ ) given as a 90-minute infusion once weekly. Four days before the second irinotecan dose, patients received $200 \mathrm{mg}$ MT capsules t.i.d. $(=600 \mathrm{mg} /$ day), for 14 consecutive days (each capsule containing $200 \mathrm{mg}$ MT seed extract, containing $80 \%$ silymarin). Short-term (4 days) or more prolonged (12 days) intake of MT in this study had no significant effect on irinotecan clearance (mean, 31.2 vs. 25.4 vs. $25.6 \mathrm{~L} / \mathrm{h} ; \mathrm{p}=0.16$ ). The AUC ratio of the active metabolite $\mathrm{SN}-38$ and the irinotecan parent drug was slightly decreased by MT ( $2.58 \%$ vs. $2.23 \%$ vs. $2.17 \%$; $=0.047$ ), whereas the relative extent of glucuronidation of $\mathrm{SN}-38$ was similar (10.8 vs. 13.5 vs. $13.1 ; p=0.64$ ). Likewise, the AUC ratio of 7ethyl-10-[4-N-(5-aminopentanoic acid)-1-piperidino]-carbonyloxycamptothecin and irinotecan was unaffected by MT $(0.332$ vs. 0.285 vs. $0.337 ; p=0.53$ ). This study showed that the concurrent use of MT does not significantly influence the PK of irinotecan in cancer patients. The authors indicated that irinotecan is known to be highly susceptible to CYP3A4 and P-gp (ABCB1) inhibition and induction in humans and concluded that the results of the current study confirm that MT does not seem to modulate the activity and/or expression of these proteins. They also noted that this finding is consistent with the notion that the MT constituent silybin is not a ligand activator of the pregnane $\mathrm{X}$ receptor (PXR), a steroid- and xenobiotic-regulated transcription factor that is a key regulator of constitutive expression and induction of CYP3A4, UGT1A, and ABCB1. The results of the irinotecan study also provide additional information in support of an apparent lack of effect of MT on the function of human carboxylesterase 2-mediated hydrolysis and UGT1A-mediated phase II conjugation pathways in humans.

There are two studies regarding the assessment of potential MT effects on the PK of more or less sensitive CYP2C9 substrates available $[58,59]$. Han and colleagues investigated the effects of silymarin on the PK of the angiotensin II (ATII) receptor antagonist losartan (50 mg) and its active metabolite E- 3174 and its possible relationship to CYP2C9 genotypes because current evidence suggests that losartan biotransformation to E-3174 is highly dependent on CYP2C9 activity [59]. Twelve healthy adult male subjects of known CYP2C9 genotype (six CYP2C9*1/*1 and six CYP2C $* 1 / * 3$ ) were enrolled, and the PK of losartan and E-3174 were assessed before and after a 14-day treatment with $140 \mathrm{mg}$ of silymarin t.i.d. ( $=420 \mathrm{mg} /$ day; Legalon $^{\circledR} 140 \mathrm{mg}$ capsules).

About $\geq 2$-fold differences in the $\mathrm{AUC}_{0-24}(409.9 \pm 76.9$ vs. $854.3 \pm 236.9 \mathrm{ng} \mathrm{h} / \mathrm{mL}$, placebo vs. silymarin, $\mathrm{p}<0.05$ ), $\mathrm{AUC}_{0-\infty}$ 
( $425.0 \pm 83.9$ vs. $886.0 \pm 221.1 \mathrm{ng} \mathrm{h} / \mathrm{mL}$, placebo vs. silymarin, $\mathrm{p}<0.05)$ and $\mathrm{C}_{\max }(170.9 \pm 54.6$ vs. $323.3 \pm 121.0 \mathrm{ng} / \mathrm{mL}$, placebo vs. silymarin, $\mathrm{p}<0.05$ ) of losartan between the placebo and silymarin treatments were observed in carriers of CYP2C ${ }^{*} 1 /{ }^{*} 1$. Accordingly, the oral clearance $(\mathrm{CL} / \mathrm{F})$ of losartan was also significantly decreased by more than 2-fold after a 14-day silymarin treatment in these subjects $(121.9 \pm 27.2$ vs. $59.7 \pm 159.7 \mathrm{~L} / \mathrm{h}$, placebo vs. silymarin, $\mathrm{p}<0.05)$. In contrast, in carriers of CYP2C $9 * 1 / * 3$, no significant differences in any of losartan PK parameters between the placebo and silymarin treatment groups were observed, e.g., $\mathrm{AUC}_{0-24}$ was $816.3 \pm 282.9$ vs. $805.8 \pm$ $266.2 \mathrm{ng} \mathrm{h} / \mathrm{mL}$, and $\mathrm{AUC}_{0-\infty}$ was $866.0 \pm 292.8 \mathrm{vs} .871 .9 \pm 23 \mathrm{ng} \mathrm{h} /$ $\mathrm{mL}$. Likewise, the metabolic ratio of losartan (ratio of $\mathrm{AUC}_{0-\infty}$ of $\mathrm{E}-$ 3174 to $\mathrm{AUC}_{0-\infty}$ of losartan) was significantly decreased after silymarin treatment in carriers of CYP2C9*1/* $1(\mathrm{p}<0.05)$, but not in those with the CYP2C9*1/*3 genotype ( $\mathrm{p}=0.065)$. Notably, the total exposure parameters (i.e., $\mathrm{AUC}_{0-24}$ and $\mathrm{AUC}_{0-\infty}$ ) in placebotreated carriers of CYP2C ${ }^{*} 1 /{ }^{*} 3$ were well comparable to silymarin-treated CYP2C9*1/*1 subjects, thereby suggesting that the extensive CYP2C9 metabolizers (i.e., carriers of CYP2C9*1/*1 wildtype) were actually subject to a silymarin-mediated pharmacological conversion to heterozygous intermediate CYP2C9 metabolizers (i.e., CYP2C9*1/*3).

As CYP2C9 appears responsible for oxidative conversion of losartan to its pharmacologically active carboxylic acid metabolite E3174 , the data of the present study are consistent with a substantial CYP2C9 inhibitory potential of silymarin treatment at customary doses in vivo. As E-3174 has a higher ATII antagonistic potency and longer half-life than the parent compound losartan, the active metabolite may be possibly responsible for most of the antihypertensive effect, and the decrease in the metabolic ratio of losartan to E-3174 due to decreased CYP2C9 activity may diminish the efficacy of losartan in carriers of CYP2C9*1/*1 [59]. The study results of Han et al. were recently followed up by Brantley and colleagues, who identified in vitro that silybin A and silybin B were the most potent CYP2C9 inhibiting constituents of silymarin extract, with $\mathrm{K}_{\mathrm{i}}$ values of 4.8 and $10 \mu \mathrm{M}$, respectively [44]. In mechanistic terms, the available CYP2C9-related evidence suggests that MT treatment may have a potential to inhibit the clearance and to increase the exposure of other sensitive CYP2C9 substrates such as (S)-warfarin in vivo.

An earlier study examined the influence of a 9-day silymarin pretreatment at a low daily dose of $140 \mathrm{mg}$ on the metabolism and disposition of metronidazole $(3 \times 400 \mathrm{mg} /$ day), a substrate of CYP3A4 and CYP2C9, in 12 healthy adult subjects [58]. In this study, low-dose silymarin modestly increased the clearance of metronidazole and its major metabolite, hydroxy-metronidazole (HM), by $29.51 \%$ and $31.90 \%$, respectively, with a concomitant decrease in half-life, $\mathrm{C}_{\max }$, and $\mathrm{AUC}_{0-48}$. However, urinary excretions of acid-metronidazole (AM), HM as well as metronidazole in $48 \mathrm{~h}$ were decreased. The authors concluded that the results indicate that silymarin might induce both intestinal P-gp and CYP3A4 upon multiple dose administration. However, this interpretation would not be consistent with all other published in vivo studies examining the effects of silymarin on CYP3A4 and P-gp function. Hence, the underlying mechanism(s) of the reported modest silymarin/metronidazole PK interaction remain(s) to be determined.

Two studies investigated the effect of MT administration on the PK of the prototypic P-gp (ABCB1) substrates digoxin [60] and the $\beta$-adrenoceptor antagonist talinolol [61], with the aim to examine potential in vivo effects of MT treatment on P-gp function.
Sixteen healthy young subjects ( $8 \mathrm{~m} / 8 \mathrm{f}$ ) were randomly assigned to receive a standardized MT supplement for 14 days (300 mg t. i. d. $=900 \mathrm{mg} /$ day, standardized to contain $80 \%$ silymarin), or to receive rifampin (300 $\mathrm{mg}$ b.i.d. $=600 \mathrm{mg} /$ day, 7 days ) and clarithromycin (500 mg b.i.d. $=1000 \mathrm{mg} /$ day, 7 days) as positive controls for P-gp (ABCB1) induction and inhibition, respectively. Oral digoxin (Lanoxicaps ${ }^{\mathrm{TM}}, 0.4 \mathrm{mg}$ ) was administered as a P-gp probe before and at the end of supplementation and control periods. Serial digoxin serum concentrations were obtained over $24 \mathrm{~h}$ [60].

As expected, rifampin produced significant reductions $(\mathrm{p}<0.01)$ in measures of total $\left(\mathrm{AUC}_{0-3}, \mathrm{AUC}_{0-24}\right)$ and maximum $\left(\mathrm{C}_{\max }\right) \mathrm{di}-$ goxin exposure, whereas clarithromycin increased these parameters significantly $(\mathrm{p}<0.01)$. In turn, no remarkable effects on digoxin PK were observed following supplementation with MT, although digoxin $\mathrm{AUC}_{0-3}$ and $\mathrm{AUC}_{0-24}$, and $\mathrm{C}_{\max }$ were altogether found modestly reduced following MT administration by about $9.5 \%, 12.5 \%$, and $13.5 \%$, respectively. These results do not support reported in vitro data suggesting a potential inhibition of P-gp function by MT constituents and also do not suggest a clinically relevant modulation of P-gp activity by MT supplementation at customary doses.

Han and coworkers examined the effect of concomitantly administered silymarin on the pharmacokinetics of talinolol, a drug mainly eliminated into the intestine by P-gp, in 18 healthy male Chinese subjects, and its association with a genetic single nucleotide polymorphism in exon 26 (C3435T) of the MDR-1 (ABCB1) gene [61], which was shown to be correlated with intestinal expression and in vivo activity of P-gp [62]. The PK of talinolol was assessed after coadministration of either placebo or $140 \mathrm{mg}$ silymarin capsules t.i.d. ( $420 \mathrm{mg} /$ day) for 14 days, and talinolol plasma concentrations were followed for 36 hours. Talinolol peak plasma concentrations $\left(C_{\max }\right)$ were significantly increased after silymarin administration as compared with placebo $(\mathrm{p}=0.007)$, and the total exposure estimates $\left(\mathrm{AUC}_{0-36}\right.$ and $\mathrm{AUC}_{0-\infty}$ ) of talinolol were increased on average by about $36 \%$ by silymarin coadministration. Accordingly, the oral clearance $(\mathrm{CL} / \mathrm{F})$ of talinolol was decreased by about $23 \%$ ( $p<0.001)$. These effects were unrelated to the MDR-1 C3435T single nucleotide polymorphism, thereby suggesting a P-gp-unrelated mechanism of this finding, probably consistent with an upregulation of intestinal organic anion transporting polypeptide (OATP) mediated uptake of talinolol [63]. Deng and coworkers examined the interaction of MT extract and the cholesterol lowering HMG-CoA-reductase inhibitor rosuvastatin [64]. Eight healthy Korean male subjects were either treated with placebo or silymarin (Legalon ${ }^{\circledR} 140 \mathrm{mg}$ capsules) t.i.d. ( $=420 \mathrm{mg} /$ day) for 5 days. Subjects received a single $10 \mathrm{mg}$ dose of rosuvastatin on day 4 of silymarin supplementation, and serial blood samples were collected for $72 \mathrm{~h}$. During plasma sampling, subjects continued to take silymarin or placebo capsules t.i.d. for 2 further days. The plasma concentration-time profile of rosuvastatin showed no significant effect of silymarin treatment. $\mathrm{C}_{\max }$ values (mean $\pm \mathrm{SD}$ ) for rosuvastatin were $13.7 \pm 4.7 \mathrm{ng} / \mathrm{mL}$ and $14.8 \pm 5.3 \mathrm{ng} / \mathrm{mL}$ in the presence and absence of silymarin, respectively. The AUC values (mean \pm SD) for rosuvastatin with and without silymarin treatment were $134.6 \pm 42.3$ and $144.0 \pm$ $38.7 \mathrm{ng} \mathrm{h} / \mathrm{mL}$, respectively. Thus, short-term silymarin treatment at customary doses did not significantly affect the PK of rosuvastatin, a multitransporter substrate, including the intestinal efflux/uptake transporters P-gp (ABCB1), BCRP (ABCG2), and OAT$\mathrm{P} 1 \mathrm{~A} 2$, and the hepatic uptake transporters OATP1B1, 1B3, and 
2B1 [65], indicating that silymarin is not a potent modulator of $\mathrm{ABC}$ and OATP transporters in vivo.

In conclusion, the available evidence from a total of 13 clinical PK interaction studies consistently indicates that marketed MT/silymarin products, given at customary or supra-therapeutic doses, do not alter the function of CYP3A4, CYP1A2, CYP2D6, and CYP2E1 enzymes, or intestinal $A B C$ and OATP transporters to a relevant extent, although the findings regarding a potential modulation of the activity of certain OATP transporters is not sufficiently detailed and entirely conclusive as yet, and deserves further investigation. Due to the total number of studies available and the high degree of consistency in overall study outcomes, the evidence in terms of a lack of interaction potential is most robust for CYP3A4 and P-gp (ABCB1). The results from a single study in irinotecan-treated cancer patients also suggest that the function of UGT1A1 and carboxylesterase 2 are unlikely to be substantially altered by MT/silymarin.

In contrast, the available evidence from one well-designed study in Chinese subjects regarding CYP2C9 [59], together with consistent in vitro data [44], strongly suggests the possibility of significant CYP2C9 inhibition by MT/silymarin. These data call for further investigation and confirmation of the clinical significance of this matter with narrow therapeutic index CYP2C9 substrates such as warfarin.

\section{Ginseng}

Several ginseng species (family Araliaceae) need to be distinguished including Panax ginseng (Asian ginseng), Panax quinquefolius (American ginseng), and Siberian ginseng (Eleutherococcus senticosus Maxim). For centuries ginseng has been used as a traditional herbal medicine in many Asian countries. Today ginseng products are consumed worldwide and rank among the most commonly used herbal products. Actually, ginseng is the 5th best-selling herb in the United States [66]. Ginseng products are advocated for many conditions, including maintenance of general health, physical and mental well-being, treatment of fatigue, weakness and mild depression, improving immune function, and for conferring antioxidant and antineoplastic pharmacological effects $[67,68]$.

Among the different principal ginseng constituents, which are derived from the dried roots of the plants, the major active components are ginsenosides, which represent a diverse group of steroidal saponins [68]. Some remarkable differences between ginseng species exist regarding the content and composition of ginsenosides, a factor that may be associated with a differential drug interaction potential. The recommended daily dose of $P$. ginseng is $200 \mathrm{mg}$ of standardized (i.e., $4 \%$ total ginsenosides) extract.

The effects of ginseng extracts or individual ginsenosides on CYP enzymes have been examined in many published in vitro studies. Unfortunately the results have been largely inconsistent and remain controversial. Also the concentrations required to elicit CYP inhibition or induction in vitro were often very high and unlikely to be achieved in vivo with recommended doses of marketed products [69]. Hence, reliable predictions on the in vivo relevance of these findings are hardly possible.

Clinical studies on PK ginseng-drug interactions are scarce; we identified a total of 9 published PK interaction studies reporting original data.

In a phenotyping study measuring single timepoint, phenotypic metabolic ratios in elderly subjects with administration of the prototypic enzyme-specific probe drugs MDZ (CYP3A4), caffeine
(CYP1A2), chlorzoxazone (CYP2E1), and debrisoquine (CYP2D6), the potential of $P$. ginseng, at a dose of $500 \mathrm{mg}$ t.i.d. $(=1.500 \mathrm{~g} /$ day, standardized to $5 \%$ ginsenosides) given for 28 days, to alter the activities of these CYP enzymes was investigated [18]. In this setting, P. ginseng did not alter the function of CYP3A4, CYP1A2, and CYP2E1, and displayed only a marginal reduction of CYP2D6 activity ( $7 \%)$, an effect magnitude not being of clinical concern. In a similar study in healthy young subjects, using the same $P$. ginseng product, dose, and treatment duration, as well as the same CYP probe drugs, no significant effects of $P$. ginseng on any CYP enzyme activity were found [17].

The findings of Gurley et al. in terms of unaltered CYP3A4 activity were confirmed by another study, in which $P$. ginseng $100 \mathrm{mg}$ doses (Ginsana ${ }^{\mathrm{TM}}$, standardized to $4 \%$ ginsenosides) were given b.i.d. ( $=200 \mathrm{mg} /$ day) for 14 days to 20 healthy male and female subjects (10 Caucasians, 10 Asians). In this study, the urinary 6$\beta$-hydroxycortisol/cortisol ratio, a marker of CYP3A enzyme induction, remained unaltered, thereby indicating that $P$. ginseng, at recommended doses, does not confer significant CYP3A induction in vivo [70].

The effects of Siberian ginseng (SG; Eleutherococcus senticosus Maxim; 485-mg capsules of standardized extract twice daily) on the activity of CYP2D6 and 3A4 were assessed in a 2-week study in 12 healthy male and female subjects [71]. Dextromethorphan (CYP2D6) and alprazolam (CYP3A4) were used as probe drugs. There were no statistically significant differences between preand post-SG treatment urinary dextromethorphan/dextrorphan metabolic ratios, indicating a lack of an SG effect on CYP2D6 enzyme activity. For alprazolam there also were no significant differences in plasma concentrations and derived PK parameters $\left(C_{\max }, t_{\max }, A U C, t_{1 / 2}\right)$ indicating that $S G$ does not significantly induce or inhibit CYP3A4. These results are consistent with the outcomes reported for $P$. ginseng preparations as detailed above and indicate that standardized extracts of SG at generally recommended doses are unlikely to alter the disposition of coadministered medications primarily dependent on the CYP2D6 or CYP3A4 pathways for their elimination [71].

The effects of American ginseng (AG; Panax quinquefolius) on the disposition of the HIV protease inhibitor and sensitive CYP3A4 substrate indinavir were assessed in 14 healthy adult male and female subjects (12 African-American and two Caucasians) [72]. Indinavir at $800 \mathrm{mg}$ doses was administered t.i.d., either alone or together with $1.0 \mathrm{~g}$ doses of AG three times daily over 2 weeks (dried whole root $500 \mathrm{mg}$ capsules). The study demonstrated that AG did not alter the PK of the CYP3A4 substrate indinavir, thereby demonstrating the absence of appreciable CYP3A4 induction or inhibition.

Lee and colleagues assessed the effects of a 2-week treatment of $200 \mathrm{mg}$ b.i.d. (= $400 \mathrm{mg} /$ day) enriched AG extract (HT1001 $100 \mathrm{mg}$ capsules; REMEMBER-fX; total ginsenoside content of $8.5 \pm 0.5 \%$ ) on the PK profile of the nucleoside reverse transcriptase inhibitor zidovudine (single 300-mg oral doses of Retrovir ${ }^{\mathrm{TM}}$ ) [73]. This study provided the first in vivo insights into the potential effects of AG on phase 2 drug metabolizing enzymes, as zidovudine is predominantly cleared through glucuronidation by UGT 2B7. The study results showed that AG does not significantly affect the formation clearance of zidovudine to its glucuronide (ratio post- to pre-AG $=1.17$; $90 \%$ confidence interval: $0.95-$ $1.45 ; \mathrm{p}=0.21$ ), total clearance (ratio $=0.97 ; 0.82-1.14 ; \mathrm{p}=0.70$ ), or total plasma zidovudine exposure $\left(\mathrm{AUC}_{0-8}\right.$ ratio $=1.03 ; 0.87$ $1.21 ; \mathrm{p}=0.77$ ). 
Jiang and colleagues investigated the effects of $P$. ginseng on the PK and PD of warfarin in 12 healthy male subjects (eight Caucasians, four Asians) [74]. A single 25-mg dose of warfarin (Couma$\operatorname{din}^{\mathrm{TM}}, 5 \times 5$-mg tablets) was administered to each subject with and without pretreatment with multiple doses of ginseng for 1 week (Golden Glow = Korean ginseng, each capsule containing extract equivalent to $0.5 \mathrm{~g}$ Panax ginseng root and $8.93 \mathrm{mg}$ ginsenosides as ginsenoside Rg1; $2 \times 0.5$-g capsules, t.i.d. = 3 g). Dosing of ginseng was continued for a further 1 week after warfarin administration. The bioanalytics comprised enantiomer-selective quantification of S-warfarin, which is predominantly metabolized to S-7-hydroxywarfarin by CYP2C9, and R-warfarin, which is metabolized by CYP3A4 and CYP1A2, thereby allowing for a separate mechanistic assessment of any potential alteration of these metabolic pathways by concomitant $P$. ginseng treatment. The study demonstrated that $P$. ginseng had no effect on the activity of CYP1A2, CYP3A4, or CYP2C9 in healthy subjects, as treatment did not affect the PK and clearance of both warfarin enantiomers in human subjects. $P$. ginseng also did not affect the apparent volumes of distribution or protein binding of warfarin enantiomers. PD endpoints of warfarin $25 \mathrm{mg}$ single doses were also assessed in this study, and it was shown that $P$. ginseng did not significantly alter blood coagulation (i.e., INR) outcomes and platelet aggregation.

The results of this study were essentially confirmed by the same group of investigators in a warfarin interaction study of similar design, and in a comparable population (12 healthy male subjects, 6 Caucasian, 6 Asian), but using a different product (Blackmores Travel Calm Ginger, Zingiber officinale, each capsule containing extract equivalent to $0.4 \mathrm{~g}$ of ginger rhizome powder) at different daily doses ( 3 capsules, $3 \times$ daily for 1 week) [26]. Similar as in the previous study, dosing of the herbal product was continued for a further week after warfarin administration. Also in this study, the PK and PD of warfarin were essentially unaltered by repeat-dose coadministration of recommended ginseng doses, thereby confirming the findings of the previous study [26]. Finally, the results of both studies $[27,75]$ were pooled and subjected to a population PK/PD modeling approach [74]. The pooled data analyses indicated that the ratio of S-warfarin's apparent clearance $(\mathrm{CL} / \mathrm{F})$ compared to control $(=$ ratio warfarin + ginseng/ warfarin alone) was $1.14 \pm 0.04$ after $P$. ginseng pretreatment, indicating that $P$. ginseng produced only a moderate increase in the $\mathrm{CL} / \mathrm{F}$ of S-warfarin. None of the two ginseng products employed in the pooled studies had a direct effect on warfarin PD [74].

In another randomized, double-blind, placebo-controlled parallel group study in healthy adult subjects, the effects of AG (P. quinquefolius, 0.5 g capsules, total ginsenoside content of $5.19 \%$ ) on the PK and PD of warfarin were studied [76]. Patients received oral warfarin (Coumadin, DuPont Pharmaceuticals), $5 \mathrm{mg}$ daily, for the first 3 consecutive days during week 1. Beginning in week 2, patients were randomly assigned to receive either oral AG, $1.0 \mathrm{~g}$, or placebo b.i.d. (=2 g/day), for 3 consecutive weeks. During week 4, all patients again received oral warfarin, $5 \mathrm{mg}$ daily, for the first 3 consecutive days. Ginseng or placebo assignment was randomized, and a total of 20 subjects were enrolled $(9 \mathrm{~m} /$ $11 \mathrm{f} ; 12$ subjects in the ginseng group, 8 subjects in the placebo group). The authors compared changes in peak INR, INR AUC, peak plasma warfarin levels, and warfarin AUCs between the ginseng and placebo groups. The peak INR was shown to decrease statistically significantly after 2 weeks of ginseng administration compared with placebo (difference between ginseng and placebo, -0.19 [95\% CI, -0.36 to -0.07$] ; \mathrm{p}=0.0012$ ). The INR area under the curve (AUC), peak plasma warfarin level, and warfarin AUC were also significantly reduced in the ginseng group as compared with the placebo group. Also, peak INR and peak plasma warfarin levels were positively correlated. The INR responses in the ginseng group were modest, albeit significantly different from the placebo group. The average group results, however, have to be interpreted with caution, as the INR data in the ginseng group were largely driven by one outlier patient displaying a high baseline INR (1.32) and a remarkably increased peak INR after warfarin administration on day 4. After ginseng administration, the peak INR of this patient declined remarkably from 5.16 to 2.75, and the corresponding $\mathrm{AUC}_{\mathrm{INR}}$ decreased from 17.46 to 11.1. Although the authors emphasize that the results of their study remain statistically significant, when this patient is excluded from analysis, it becomes evident, that parallel group studies of such a small sample size are difficult to interpret.

Taken together, the available published evidence from clinical ginseng/warfarin interaction studies indicate that high repeated doses of $P$. ginseng and ginger over 1 week did not significantly alter the PK or PD of $25 \mathrm{mg}$ warfarin single doses in healthy adult subjects $[27,74,75]$. In contrast, a small parallel group study in healthy subjects using a different warfarin posology (5 mg warfarin q.d. on 3 consecutive days) and a 2-week treatment with $\mathrm{AG}(P$. quinquefolius) at the high end of the recommended dose range suggests that AG may reduce warfarin exposure (i.e., increases warfarin clearance), thereby reducing the anticoagulant effect of warfarin to a modest extent. The differences in the outcome of these studies might be either due to differential properties of $P$. ginseng and AG (P. quinquefolius) products, differences in the study designs (1-week vs. 2-week treatments), or simply due to the limitations of the small parallel group study which was apparently confounded by an outlier subject [76].

While all ginseng/warfarin interaction studies available so far suffer the principle design limitation of short-term warfarin treatments in healthy adult subject populations, which may not be predictive for long-term warfarin treatment in typical patient populations, the totality and consistency of data derived from mechanistic PK studies as detailed above, strongly suggest a lack of relevant CYP enzyme inhibitory or induction properties of ginseng products at recommended therapeutic doses.

As yet, no clinical studies on the investigation of ginseng products regarding their capacity to confer transporter-based interactions have been published. Therefore, the available published evidence from clinical trials suggests an overall low potential of virtually all ginseng species for CYP-based ginseng-drug interactions, while clinical studies on transporter-based interactions are still lacking.

\section{Goldenseal}

Goldenseal (Hydrastis canadensis, orangeroot) is a perennial herb in the buttercup family Ranunculaceae, indigenous to southeastern Canada and the northeastern United States. Botanical supplement goldenseal (GS) preparations are taken for the prevention and treatment of the common cold, upper respiratory and gastrointestinal infections, as well as menstrual disorders. In addition, GS is being used as a topical antimicrobial remedy to disinfect cuts and scrapes. GS contains a number of isoquinoline alkaloids such as hydrastine, berberine, berberastine, hydrastinine, tetrahydroberberastine, canadine, and canalidine. Hydrastine and berberine are the main active constituents [77].

As a supplement, standardized extracts of GS are available in liquid, tablet, and capsule forms. Recommended doses of the pow- 
dered root vary widely and range from $750 \mathrm{mg}$ to 6 grams in tablet or capsule form per day, to be given 2 to 3 times daily. GS preparations enjoy widespread popularity and are among the top 20 bestselling botanical products in the US [78].

A total of 8 published pharmacokinetic in vivo drug interactions with orally administered GS root extract ( 5 studies) or berberine products ( 3 studies) were identified.

In the first published study, the influence of GS root extract (GS root herbal single 570-mg capsules, two capsules b.i.d.= $2.280 \mathrm{mg} /$ day, given for 14 days) on the disposition of the HIV protease inhibitor as well as CYP3A4 and P-gp substrate indinavir was examined in 11 healthy subjects ( $6 \mathrm{~m} / 5 \mathrm{f})$ [79]. Indinavir was given as a single 800 -mg oral dose; blood samples were collected for 8 hours postdose, and the PK of indinavir was characterized before and after 14 days of GS treatment. No statistically significant alterations in the PK of indinavir were observed (AUC values not reported). The oral clearance $(\mathrm{CL} / \mathrm{F})$ of indinavir was lowered on average by $4.6 \%$ following GS treatment, with individual changes ranging from $-46.1 \%$ (subject 5 ) to a $46 \%$ increase (subject 4), indicating a remarkable intersubject variability in this study. Accordingly, the $95 \% \mathrm{CI}$ for the difference in oral clearance between treatments was large ( -10.6 to $3.4 \mathrm{~L} / \mathrm{h}$ ). Similar changes were observed in $\mathrm{C}_{\max }$, with the $95 \% \mathrm{CI}$ for the difference in peak concentration with goldenseal root ranging from -0.6 to $2.5 \mathrm{mg} /$ L. Overall, the study results suggest that GS supplementation at customary doses appears unlikely to affect the activity of CYP3A4 and/or P-gp (ABCB1) to a relevant extent in vivo, although the remarkable intersubject variability observed may pose questions regarding the robustness and reliability of the study. The authors also discussed the possibility of a limited study sensitivity regarding inhibition of presystemic CYP3A4, as indinavir was previously shown in a grapefruit juice interaction study to be a poor probe substrate for assessing CYP3A4-mediated gut wall extraction [80].

In a subsequent study, single timepoint, metabolic ratios of CYP probe drugs were used in 12 healthy subjects $(6 \mathrm{~m} / 6 \mathrm{f}$, extensive metabolizers of CYP2D6) to determine whether a 28 day supplementation of GS root extract $(900 \mathrm{mg}$ t.i.d. $=2.700 \mathrm{mg} /$ day; no standardization claim) affects CYP1A2, CYP2D6, CYP2E1, or CYP3A4/5 activity [8]. Probe drug cocktails of MDZ (CYP3A4) and caffeine (CYP1A2), followed 24 hours later by chlorzoxazone (CYP2E1) and debrisoquine (CYP2D6) were administered before (baseline) and at the end of supplementation. Presupplementation and postsupplementation assessments of CYP3A4, CYP1A2, CYP2E1, and CYP2D6 activities were made by use of 1-OH-MDZ/ MDZ serum ratios (1-hour sample), paraxanthine/caffeine serum ratios (6-hour sample), 6-hydroxychlorzoxazone/chlorzoxazone serum ratios (2-hour sample), and debrisoquine urinary recovery ratios (8-hour collection), respectively.

Comparisons of pre- and postsupplementation metabolic ratios indicated significant inhibition of about 40\% of CYP2D6 [geom. mean post-/pre-ratio $(90 \% \mathrm{CI})$ of $0.60(0.51$ to 0.70$)]$ and CYP3A4/5 [geom. mean post/pre ratios ( $90 \% \mathrm{CI}$ ) of 0.60 (0.53 to 0.67$)$ ] activities due to GS supplementation, while there was no appreciable effect on CYP1A2 and CYP2E1 activities. Hence, this was the first clinical study strongly suggesting that GS root extract, given at recommended doses, appeared to inhibit CYP2D6 and CYP3A4/5 to a notable extent in vivo.

For CYP3A4/5, the same group of authors confirmed this first clinical indication by a more comprehensive PK interaction study that comprised the assessment of complete plasma concentration-time profiles of the respective probe drugs [9]. In this study, the effects of GS supplementation (GS root extract $1.323 \mathrm{mg}$ t.i.d. $=3.969 \mathrm{mg} /$ day, standardized to contain $24.1 \mathrm{mg}$ isoquinoline alkaloids per capsule) on human CYP3A activity were evaluated using MDZ as a phenotypic probe. Sixteen healthy young subjects ( $8 \mathrm{~m} / 8 \mathrm{f}$ ) were assigned to receive goldenseal for 14 days. Oral MDZ ( $8 \mathrm{mg}$ ) was administered before and after goldenseal supplementation, and serum concentrations of MDZ and the main metabolite 1-OH-MDZ were followed up to 6 hours postdose. In order to demonstrate the sensitivity of the study to adequately capture any relevant effects on CYP3A4 activity, the wellestablished CYP3A4 inducing/inhibiting probe drugs rifampin (300 mg b.i.d. for 7 days) and clarithromycin (500 mg b.i.d. daily for 7 days) were employed in the study as well. Comparisons of pre- and post-GS supplementation MDZ PK parameters indicated significant inhibition of CYP3A by GS $\left(\right.$ AUC $_{0-\infty} 107.9 \pm 43.3$ vs. $175.3 \pm 74.8 \mathrm{ng} \cdot \mathrm{h} / \mathrm{mL} ; \mathrm{Cl} / \mathrm{F} / \mathrm{kg}, 1.26 \pm 0.59$ vs. $0.81 \pm 0.45 \mathrm{l} / \mathrm{h} / \mathrm{kg}$; $\mathrm{T}_{1 / 2} 2.01 \pm 0.42$ vs. $3.15 \pm 1.12 \mathrm{~h} ; \quad \mathrm{C}_{\max } 50.6 \pm 26.9$ vs. $71.2 \pm$ $50.5 \mathrm{ng} / \mathrm{mL}$ ). Comparisons of these data show that mean MDZ $\mathrm{AUC}_{0-\infty}$ and $\mathrm{C}_{\max }$ data were increased by about $63 \%$ and $40 \%$, respectively, upon GS supplementation. In contrast, the potent CYP3A4 inhibitor clarithromycin produced about 5.5-fold (i.e., $548 \%$ ) and about 2-fold (217\%) increases in $\mathrm{MDZ} \mathrm{AUC}_{0-\infty}$ and $\mathrm{C}_{\max }$ outcomes, respectively. Taken together, these data categorize GS root extract, at the given dose, as a weak inhibitor of CYP3A4 (i.e., <2-fold increase in AUC of the sensitive CYP3A4 probe MDZ), according to current FDA drug interaction guideline recommendations [81].

In a follow-up study addressing GS potential to inhibit CYP2D6, the effects of a 14-day treatment with standardized GS root extract $(1.070 \mathrm{mg}$, standardized to contain $24.1 \mathrm{mg}$ isoquinoline alkaloids per capsule, t.i.d. $=3.210 \mathrm{mg} /$ day) on the activity of CYP2D6 was investigated in 16 healthy subjects $(8 \mathrm{~m} / 8 \mathrm{f})$, after administration of the CYP2D6 substrate debrisoquine $(5 \mathrm{mg})$ before and at the end of supplementation, by using 8-h debrisoquine urinary recovery ratios [53]. Comparisons of pre- and postsupplementation ratios indicated a significant inhibition of CYP2D6 activity by GS supplementation by about 47\% (post/pre geom. mean ratio $0.53,90 \% \mathrm{CI} 0.44$ to 0.64 ), thereby confirming previously reported single-point phenotyping data [18]. Quantitatively, these data categorize GS root extract, at the given dose, as a weak inhibitor of CYP2D6 (i.e., $<50 \%$ decrease in substrate clearance) according to current FDA drug interaction guideline recommendations.

To examine potential effects of GS root extract supplementation on P-gp (ABCB1) function in vivo, 20 healthy young subjects $(10 \mathrm{~m} / 10 \mathrm{f})$ were randomly assigned to receive a standardized GS supplement $(1.070 \mathrm{mg}$ t.i.d. $=3.210 \mathrm{mg} /$ day $)$ for 14 days or to receive the established positive control probe drugs rifampin (300 mg b.i.d. for 7 days) and clarithromycin ( $500 \mathrm{mg}$ b.i.d. for 7 days). Supplementation/medication phases were separated by 30-day washout periods. Digoxin (Lanoxin ${ }^{\mathrm{TM}}, 0.5 \mathrm{mg}$ ) was administered p.o. before and at the end of each supplementation/medication period, and serial digoxin serum concentrations were determined over $24 \mathrm{~h}$ postdose [82].

Statistically significant increases $(\mathrm{p}<0.001)$ in digoxin total and maximum exposure parameters $\left[\mathrm{AUC}_{0-24}(57 \%), \mathrm{AUC}_{0-3}(83 \%)\right.$, $\left.\mathrm{C}_{\max }(95 \%)\right]$, and terminal disposition half-life (79\%) were observed after 7 days of clarithromycin treatment. Accordingly, clarithromycin produced a 53\% decrease in $C L / F(p<0.001)$. In turn, statistically significant reductions $(\mathrm{p}<0.05)$ in digoxin $\mathrm{AUC}_{0-24}(-16 \%), \mathrm{AUC}_{0-3}(-27 \%)$, and $\mathrm{C}_{\max }(-28 \%)$ were noted following rifampin administration. Taken together, the results of 
the P-gp probe inhibitor/inducer indicate adequate sensitivity of the study to accurately capture relevant effects on P-gp function. As a result of GS supplementation, there were no significant changes in digoxin PK observed, apart from a 14\% increase in digoxin $\mathrm{C}_{\max }(\mathrm{p}<0.05)$. Based on the results of this study, it can be concluded that GS root extract supplementation at daily doses of about $3 \mathrm{~g}$ does not modify P-gp function to a clinically relevant extent.

Two studies examined the effect of berberine, a major alkaloid constituent in GS extract, on the PK of cyclosporine A (CsA) in Chinese renal transplant patients [83] and in healthy adult Chinese subjects [84].

In a randomized, controlled clinical trial in 104 renal transplant recipients, $\mathrm{Wu}$ and colleagues examined the effects of oral berberine supplementation [BBR hydrochloride tablets $200 \mathrm{mg}$ (unspecified source), t.i.d. for 3 months] on the PK of CsA [83]. Patients were randomly assigned in a $1: 1$ fashion to a BBR-free group or to the BBR treatment group. Blood trough concentration of CsA and markers for hepatic and renal functions were determined. For a more comprehensive PK substudy, six renal transplant recipients were treated with a $3-\mathrm{mg} / \mathrm{kg}$ dose of CsA b.i.d. before and after oral coadministration of $200 \mathrm{mg}$ BBR t.i.d. for 12 days.

In the clinical study, the final CsA trough concentrations and the ratios of concentration/dose of CsA in BBR-treated patients were modestly increased, i.e., $29.3 \%$ and $27.8 \%$ higher than those in BBR-free patients, respectively $(\mathrm{p}<0.05)$. In the dedicated PK study in 6 patients, the average increase in the total CsA exposure (AUC) upon BBR treatment was 34.5\% $(\mathrm{p}<0.05)$, while average increases in $C_{s s}$ and $C_{\min }$ values of CsA were $34.5 \%$ and $88.3 \%$, respectively, without significant alterations in $C_{\max }$. The average decrease in $\mathrm{CL} / \mathrm{F}$ was $40.4 \%$. Overall, the study results suggest a weak inhibition of CYP3A4 by BBR supplementation at a $600 \mathrm{mg} /$ day dose level.

In the second BBR/CsA interaction study, the effects of $300 \mathrm{mg}$ $B B R$, b.i.d. (= $600 \mathrm{mg} /$ day), given for 10 days on the pharmacokinetics of CsA were examined in 12 healthy male Chinese subjects. CsA PK assessments at a dose of $6 \mathrm{mg} / \mathrm{kg}$ were done in 6 subjects both before and at the end of the BBR treatment period, whereby the second CsA dose was given $\geq 12 \mathrm{~h}$ after the last BBR dose. Another 6 subjects received $3 \mathrm{mg} \mathrm{CsA} / \mathrm{kg}$, the second CsA dose being given concomitantly with a single oral dose of $300 \mathrm{mg} B B R$. In the study with $6 \mathrm{mg} \mathrm{CsA} / \mathrm{kg}$ treatment and time-separated intake of the last BBR dose, BBR did not significantly alter the PK of CsA. However, in the trial with $3 \mathrm{mg} \mathrm{CsA} / \mathrm{kg}$ and the concomitant administration of CSA and BBR, a modest average increase in the AUC of CsA of $19.2 \%$ ( $p<0.05$ ) was observed, without any accompanying changes in the terminal disposition half-life or apparent oral clearance $(\mathrm{CL} / \mathrm{F})$ of $\mathrm{CsA}$. These results indicate that BBR can modestly increase exposure of CsA, when both drugs are administered together [84].

Taken together, although not entirely consistently, both BBR/CsA studies are suggestive for a modest BBR-mediated inhibition of CYP3A4, which may need to be considered in case of concomitant treatment with narrow therapeutic index drugs that are sensitive substrates of CYP3A4, such as CsA.

In the most recent study, 18 Chinese healthy male adult subjects received orally either placebo or berberine (BBR) capsules at a dose of $300 \mathrm{mg}$ t.i.d. (= $900 \mathrm{mg} /$ day) for 14 days, and phenotypic effects of BBR supplementation on the activities of CYP1A2, CYP2C9, CYP2C19, CYP2D6, and CYP3A4 were examined by the administration of the probe substances caffeine (93 $\mathrm{mg}$ ), losartan
(30 g), omeprazole (20 mg), dextromethorphan (30 mg), and MDZ (2 mg), respectively [10]. The PK profiles of omeprazole, MDZ, and caffeine were assessed over $12 \mathrm{~h}$ postdose, and the losartan/ E-3174 as well as dextromethorphan/dextrorphan urinary ratios were assessed by urine sampling over $8 \mathrm{~h}$ postdose.

A remarkable decrease in CYP2D6 activity was observed in this study as the $0-8 \mathrm{~h}$ urinary dextromethorphan/dextrorphan ratio increased about 9-fold ( $p<0.01$ ). In addition, the losartan/E-3174 ratio doubled $(\mathrm{p}<0.01)$ after BBR administration, indicating a notable decrease in CYP2C9 activity. In contrast, CYP3A4 activity was only modestly inhibited, as the $C_{\max }, A C_{0-\infty}$, and $\mathrm{AUC}_{0-12}$ of MDZ were increased 38\% ( $p<0.05), 40 \%(\mathrm{p}<0.01)$, and $37 \%$ $(\mathrm{p}<0.05)$, respectively, after BBR treatment. Accordingly, the oral MDZ clearance was also modestly decreased by $27 \%(\mathrm{p}<0.05)$. There were no significant alterations of the probe substances caffeine (CYP1A2) and omeprazole (CYP2C19). Taken together, repeated BBR administration (900 mg/day) decreases CYP2D6 activity significantly, appears to modestly modify 2C9 and CYP3A4 activities, and does not alter CYP1A2 and CYP2C19 function.

In conclusion, 5 pharmacokinetic interaction studies with different GS root extract products at daily doses up to $4 \mathrm{~g}$ are overall largely consistent and characterize GS root extract as a weak inhibitor of CYP3A4 and CYP2D6 enzymes, when FDA drug interaction guideline criteria are considered. The available evidence for this assessment is most robust for CYP3A4, and based on phenotypic serum ratios or metabolite urinary recovery data only, for CYP2D6. Results from single studies indicate that GS root extract does not alter the activities of CYP1A2 and CYP2E1 enzymes, and the efflux transporter P-gp (ABCB1) to an appreciable extent. No information is available regarding potential effects of GS on CYP2C9 and CYP2C19 enzymes.

The available in vivo evidence on the PK drug interaction potential of BBR products is derived from 3 studies, altogether conducted in Chinese subjects. These studies consistently point to a weak potential of BBR products to inhibit CYP3A4. In addition, a single phenotyping study suggests that BBR decreases CYP2D6 activity and modestly modifies CYP2C9 function, but is unlikely to alter CYP1A2 and CYP2C19 functions. These findings and their clinical relevance, however, need to be confirmed by future studies. So far, caution should be applied when GS root extract or BBR products are coadministered with narrow therapeutic index drugs, which are sensitive substrates of CYP3A4, CYP2D6 or CYP2C9 enzymes.

\section{Garlic}

Garlic (Allium sativum; family Amaryllidaceae) has been cultivated and used for the alleviation of a variety of medical conditions for thousands of years. Today, garlic is worldwide still one of the most popular and most commonly used herbal supplements with purported antioxidative, antibacterial, antiparasitic, antilipidemic, antihypertensive, antiatherosclerotic, antithrombotic, antidiabetic, immune-stimulating and anticancer properties [85-87].

Pharmaceutical products derived from garlic bulbs/cloves are provided in the forms of powders, oily preparations, or aqueousalcoholic extracts of fresh or aged garlic. Due to the complex chemistry, differing manufacturing processes result in preparations of quite different chemical composition and pharmaceutical properties, which in turn may result in different pharmacological, safety, and bioavailability characteristics $[12,88]$.

The so far identified active constituents of garlic include alliin, allinase, diallyldisulfide, ajoens, and others. Alliin is enzymatically 
converted to allicin, the major garlic component, which, however, displays considerable chemical instability and is rapidly degraded to a variety of organosulfur compounds (OSCs such as diallyl sulfide, diallyl disulfide, diallyl trisulfide) that are believed to ultimately confer the main pharmacological effects [89].

Recommended doses range around $4 \mathrm{~g}$ of fresh garlic daily, which is equivalent to approximately $8 \mathrm{mg}$ garlic oil or 600 to $900 \mathrm{mg}$ garlic powder preparations standardized to $1.3 \%$ alliin content [89].

Despite the widespread use of garlic preparations along with reported preclinical evidence and anecdotal clinical case reports on the potential role of garlic in metabolism-based PK interactions and also some susceptibility for PD interactions, well-designed clinical studies evaluating drug interactions with garlic preparations in a systematic fashion are notably very limited.

Nonclinical studies suggest that garlic components may exert enzyme inducing effects on CYP1A1/2 and CYP2B1/2 family members via CAR and Nrf2 activation [90], and also to strongly induce GST activity [91], whereby upregulation of GST has been implicated in the detoxification of carcinogens. In turn, inhibition of CYP2E1, CYP2C9, and 2C19 by garlic and QSCs was suggested by preclinical data [69].

Unfortunately, the clinical relevance of these findings for commonly employed human doses has not been completely followed up as yet by well-designed mechanistic in vivo studies for many of the described metabolic pathways. Hence, based on currently published evidence, the question of clinical relevance for the described effects on CYP2B1/2 family members and UGTs, GST, QR, and EH enzymes remains virtually still unaddressed to the best of our knowledge.

A total of 8 studies in healthy adult young and elderly subjects using different garlic products (600 mg garlic tablets, 3 tablets b.i.d. [92]; garlic oil $500 \mathrm{mg}$, t.i. d. [18, 19], garlic oil extract, diallyl sulphide (DAS) [93]) and study designs have so far addressed various aspects of the PK drug interaction potential of garlic treatment. Apparently, the most recent human in vivo study addressing garlic-related PK drug interaction issues was published in 2005 , thereby indicating some stagnation rather than ramp-up of clinical activities in this area of research.

A total of 3 interaction studies with administration of prototypic enzyme-specific probe drugs assessed the potential of various garlic preparations to alter activities of CYP2E1 [93], CYP2D6 and 3A4 [92], CYP1A2, CYP2D6, CYP2E1, or CYP3A4 [17] in healthy subjects, and one study used a similar approach to probe for alterations of CYP1A2, CYP2D6, CYP2E1, and CYP3A4 activities in elderly individuals [18].

These studies indicate consistently that neither single nor repeat dose administrations of various garlic formulations up to 28 days may have potential to exert notable alterations (i.e., inhibition or induction) of CYP1A2, CYP2D6, or CYP3A4 activities in humans, which are important metabolic pathways for many drugs likely to be coadministered with garlic supplements. In clinical terms, these results indicate that garlic preparations are unlikely to alter the disposition of coadministered drugs that are sensitive substrates of these oxidative metabolic pathways.

In contrast, repeat administration of garlic oil over 28 days was shown to modestly reduce CYP2E1 activity [estimated by 6- hydroxychlorzoxazone (6-OH-CZX)/chlorzoxazone (CZX) metabolic ratio] by $39 \%$ in healthy adult subjects [17] and by approximately $22 \%$ in elderly individuals (mean age 67 years) [18]. These results are consistent with an earlier study on single dose effects of $0.2 \mathrm{mg} / \mathrm{kg}$ BW garlic oil extract, diallyl sulphide (DAS), on CYP2E1 activity, which also employed the 6-OH-CZX/CZX metabolic ratio for the estimation of CYP2E1 function [93]. However, as the overall extent of CYP2E1 inhibition by garlic is modest, i.e., conceptually metabolic ratios always display quantitatively greater changes than respective clearance alterations of the prototypic substrate, and hardly any currently approved drugs in widespread use are relying on CYP2E1 as a sole or predominant clearance pathway (except chlorzoxazone), the observed CYP2E1 effects of garlic do not appear to have important clinical implications. This perspective is further supported by a study in healthy adult subjects, in which a 3-month administration of aged garlic extract (10 mL q.d.) did not show any notable effect on oxidative metabolism of acetaminophen, to which CYP2E1 is known to be a significant contributor, besides CYP3A4 and CYP1A2 [94]. Interestingly, in this study also no effects of garlic on GST activity could be noted, as no changes in the excretion of three acetaminophen metabolites arising from GST-mediated glutathione conjugation with the reactive acetaminophen metabolite were observed [94]. This finding casts doubts on the human in vivo relevance of the consistently reported inducing effects of garlic components on GST activity from animal and in vitro studies.

The lack of a 3-week garlic treatment (GarliPure ${ }^{\mathrm{TM}} 1.200 \mathrm{mg} /$ day) on the functional activity of intestinal and hepatic CYP3A4 in healthy adult subjects was further confirmed by demonstration of an unaltered PK of the HIV-protease inhibitor saquinavir, a sensitive CYP3A4 substrate which undergoes extensive CYP3A4mediated presystemic gut wall metabolism, and by means of an erythromycin breath test, a noninvasive marker of hepatic CYP3A4 function [95].

These findings are also in line with another healthy subject study demonstrating that a 4-day short-term treatment with garlic capsules (10 mg garlic extract q.d., provided as $2 \times 5$-mg liquidfilled soft gelatin capsules, equivalent to $1 \mathrm{~g}$ of fresh garlic) did not significantly alter the single dose PK of ritonavir, another HIV-protease inhibitor that is mainly metabolized by CYP3A4. However, the authors cautioned that in view of the complex time-dependent effects of ritonavir on drug metabolizing enzymes and transporters (ritonavir is also a potent inhibitor of CYP3A4 and the ABC efflux transporter P-gp), the results of the study may be difficult to interpret and should not be extrapolated to steady-state conditions [96].

The only study reporting a PK alteration of the HIV-protease inhibitor saquinavir upon a 3-week garlic treatment (GarliPure ${ }^{\mathrm{TM}}$ caplets b.i.d.) of ten healthy subjects suggested about $51 \%, 49 \%$, and $54 \%$ reduced mean saquinavir steady-state exposure (AUC $\tau$ ), trough levels $\left(C_{\text {trough }}\right)$, and maximum concentrations $\left(C_{\max }\right)$, respectively [55]. However, most notably, after a 10-day washout period of garlic treatment, the saquinavir AUC, trough, and $C_{\max }$ values did not return to baseline, but still remained at $60-70 \%$ of their baseline values. As the study employed a fixed sequence rather than a randomized crossover design, and in light of published evidence on time-dependent saquinavir PK characteristics in healthy subjects [97] and HIV patients [98], it is most conceivable that the study results merely mirror saquinavir-mediated induction processes of its own metabolism and disposition rather than indicating a relevant garlic-mediated drug interaction.

Taken together, there is well-consistent evidence from a variety of mechanistic PK studies that common doses of various kinds of garlic preparations do not exert any notable alteration of CYP3A4 function in vivo.

Whether or not garlic may be involved in transporter-based drug interactions has apparently not yet been addressed in mechanis- 
tic human PK studies, to the best of our knowledge. Also the preclinical evidence in this respect is less well documented [69].

Taken together, from the limited available evidence of human in vivo drug interaction studies, there is currently no indication that garlic may confer any notable or clinical significant metabolismbased PK interactions with drugs cleared by CYP1A2, CYP2D6, CYP2E1, or CYP3A4 enzymes. In contrast, unfortunately, no published evidence from human in vivo studies is currently available for the characterization of the potential effects of garlic on CYP2B family members and UGT, GST, QR, and EH enzymes or drug transporter proteins. Hence, translation of published in vitro findings into clinical interaction studies is still largely incomplete.

\section{Echinacea}

Echinacea is a genus of herbaceous flowering plants (purple coneflowers, family Asteraceae), overall comprising nine species, which are native and endemic to eastern and central North America. Marketed and studied medicinal products are made of three different species (E. purpurea, E. angustifolia, E. pallida), whereby the majority (i.e., about $80 \%$ ) of the products are based on E. purpurea. Different parts of the plants (aerial parts and roots) are used, and different pharmaceutical preparations exist (extracts and expressed juice). The German Monograph issued by the E Commission recommends alcoholic extracts from the root of E. pallida and juices pressed from the aerial parts of the plants [99]. However, the diversity in the overall composition and contents of pharmaceutical Echinacea products is considerable and may contribute to some across study inconsistencies.

Echinacea is considered to be an unspecific immunostimulant and is primarily used for the prevention and treatment of acute virus infections of the upper respiratory tract, such as the common cold and influenza. Dose recommendations for Echinacea products vary widely and depend on the formulation and concentration of the product. Recommended daily doses for adult subjects range between 900 to $1000 \mathrm{mg}$, to be given in 3 to 4 divided doses.

Despite the popularity and widespread use of Echinacea preparations and their pharmaceutical diversity, well-designed clinical studies evaluating PK drug interactions with different Echinacea preparations are notably very limited. Overall, we identified only 7 clinical PK interaction studies of Echinacea products in the public domain, which primarily addressed CYP- and P-gp-based interaction issues.

The effect of Echinacea (E. purpurea root) on CYP activities in vivo was assessed by use of single dose administrations of the CYP probe drugs caffeine (CYP1A2), tolbutamide (CYP2C9), dextromethorphan (CYP2D6), and midazolam (CYP3A) in 12 healthy subjects ( $6 \mathrm{~m} / 6 \mathrm{f})$ [100]. The probe substances caffeine $(200 \mathrm{mg})$, tolbutamide $(500 \mathrm{mg})$, dextromethorphan $(30 \mathrm{mg})$, and oral and intravenous midazolam (MDZ; $0.05 \mathrm{mg} / \mathrm{kg}$ BW i.v., and $5 \mathrm{mg}$ per oral) were administered before and after a short course of Echinacea [E. purpurea root extract $400 \mathrm{mg} 4$ times daily $(=1600 \mathrm{mg}$ / day) for 8 days], and respective plasma concentration-time profiles of the probe substances were assessed.

The disposition (i.e., $\mathrm{AUC}_{0-\infty}$, oral clearance, $\mathrm{t}_{1 / 2}$ ) of the CYP2D6 probe dextromethorphan in CYP2D6 genotyped subjects (11 CYP2D6 EMs, 1 CYP2D6 PM) was not significantly altered by Echinacea. Likewise, the ratio of the dextromethorphan/dextrorphan $\mathrm{AUC}_{0-\infty}$ values and the dextromethorphan-to-dextrorphan urinary metabolic ratio were not significantly affected by Echinacea coadministration.
The oral clearance of the CYP2C9 probe drug tolbutamide was significantly, albeit modestly, reduced on average by $11 \%$ after Echinacea dosing, with two individuals $(1 \mathrm{~m} / 1 \mathrm{f})$ displaying a $25 \%$ or greater reduction in the oral clearance of tolbutamide. In line with this, the geometric mean total tolbutamide exposure $\left(\mathrm{AUC}_{0-\infty}\right)$ before and after Echinacea dosing was modestly increased by $14 \%$ (90\% Cl 9-20\%). The maximum tolbutamide concentration was not significantly altered by Echinacea.

Echinacea dosing significantly reduced the mean oral clearance of the CYP1A2 probe caffeine by $27 \%$, with considerable intersubject variability ( $>50 \%$ in 2 individuals). Maximum serum concentrations of caffeine after a 200-mg oral dose were on average increased by $30 \%$, and the geometric mean total caffeine exposure $\left(\right.$ AUC $_{0-\infty}$ ) was increased by $29 \%$ after Echinacea dosing.

The systemic clearance of intravenously administered CYP3A4 probe MDZ was significantly increased by $42 \%$, and there was a corresponding significant decrease in mean $\mathrm{AUC}_{0-\infty}$ of $23 \%$, together suggesting that hepatic CYP3A activity was increased. In contrast, the oral clearance and the $\mathrm{AUC}_{0-\infty}$ after oral MDZ dosing were not significantly altered by Echinacea administration. However, the mean absolute oral bioavailability (F) of MDZ was increased from $24 \%$ to $36 \%$ (i.e., by $50 \%$ ), consistent with the inhibition of intestinal CYP3A by Echinacea.

The authors discussed that the differential effects of Echinacea on intestinal and hepatic CYP3A activity may be due to a variety of possible mechanisms such as locally acting CYP3A inhibiting constituents of Echinacea that do not become systemically available, or rapid absorption of CYP3A4 inducing components of Echinacea, thereby limiting intestinal exposure and intestinal CYP3A induction, or a systemically formed metabolite of a constituent of Echinacea capable of inducing hepatic CYP3A but not intestinal CYP3A. However, these mechanistic considerations remain speculative and deserve further investigation.

Taken together, the study showed that an 8-day treatment with relatively high doses of E. purpurea root extract (i.e., $400 \mathrm{mg} 4$ times daily $=1600 \mathrm{mg} /$ day) displayed differential effects on the activity of various CYP enzymes. There were no alterations of CYP2D6 noted, negligible to modest inhibition of CYP2C9, modest inhibition of CYP1A2, and differential (i.e., inductive/inhibitory) effects on hepatic and intestinal CYP3A4.

Although the observed mean effects on CYP1A2 and CYP2C9 activities were generally modest, they displayed some more pronounced effect sizes in individual subjects. Considering CYPbased Echinacea-drug interactions of potential clinical significance, the study suggests that the bioavailability of orally administered CYP3A4 substrates with low oral bioavailability may be significantly increased by Echinacea coadministration, and that the exposure to CYP1A2 and 2C9 substrates (e.g., theophylline, clozapin) may be modestly increased, at least in individual subjects. The observed effects of high doses of $E$. purpurea root extract on CYP1A2 and CYP2C9, however, are overall small and therefore unlikely to be of clinical significance in clinical practice, unless substrates with a narrow therapeutic index are concerned [e.g., (S)-warfarin in case of CYP2C9 and theophylline in case of CYP1A2].

In another study, 12 healthy subjects $(6 \mathrm{~m} / 6 \mathrm{f})$ were randomly assigned to receive E. purpurea ( $800 \mathrm{mg}$ b.i.d., no standardization claim) for 28 days [50]. Probe drug cocktails of MDZ (CYP3A4) and caffeine (CYP1A2), followed 24 hours later by chlorzoxazone (CYP2E1) and debrisoquine (CYP2D6) were administered before (baseline) and at the end of supplementation. Presupplementation and postsupplementation assessments of CYP3A4, CYP1A2, 
CYP2E1, and CYP2D6 activities were made by use of 1-OH-MDZ/ MDZ serum ratios (1-hour sample), paraxanthine/caffeine serum ratios (6-hour sample), 6-hydroxychlorzoxazone/chlorzoxazone serum ratios (2-hour sample), and debrisoquine urinary recovery ratios (8-hour collection), respectively.

In this study, high-dose E. purpurea given over 28 days did not significantly change the activities of CYP3A4, CYP2E1, and CYP2D6 as estimated by single timepoint metabolic ratios. The only perceptible change in mean phenotypic ratios occurred with 6-hour paraxanthine/caffeine values, where an approximate $13 \%$ decrease (from $0.514 \pm 0.181$ to $0.447 \pm 0.190$ ) pointed to at a possible inhibitory effect of $E$. purpurea on CYP1A2. This minor difference, however, was not statistically significant $(p=0.07)$, nor was it considered clinically relevant.

The effects of supplementation of a standardized E. purpurea extract $(267 \mathrm{mg}$ t.i.d. $=801 \mathrm{mg} /$ day, each capsule standardized to contain $2.2 \mathrm{mg}$ isobutylamides; Gaia Herbs, Inc.) on the activity of CYP2D6 was investigated in 16 healthy subjects $(8 \mathrm{~m} / 8 \mathrm{f})$ after administration of the CYP2D6 substrate debrisoquine $(5 \mathrm{mg})$ before and at the end of supplementation, by using 8-h debrisoquine urinary recovery ratios [53]. Respective results did not indicate significant inhibition of CYP2D6 by E. purpurea supplementation.

Two studies examined the effects of Echinacea treatment of the PK of two different protease inhibitor/ritonavir combination products. The most recent study was conducted in 15 HIV-infected patients ( $14 \mathrm{~m} / 1 \mathrm{f}$, median age $49 \mathrm{yrs}$, range 43 to $67 \mathrm{yrs}$ ) receiving antiretroviral therapy including darunavir-ritonavir (600/100 mg b.i.d.) for at least 4 weeks. The patients were concomitantly treated with E. purpurea root extract capsules ( $500 \mathrm{mg}$ every $6 \mathrm{~h}$ ) for 14 days on top of their antiretroviral treatment [101]. The HIV protease inhibitor darunavir undergoes extensive metabolism by CYP3A4, while ritonavir is a potent inhibitor of CYP3A4 and P-gp, and is combined with darunavir to boost (i.e., increase) its systemic exposure. Darunavir and ritonavir plasma concentrations were determined up to $12 \mathrm{~h}$ after a morning dose of darunavir-ritonavir on days 0 (darunavir-ritonavir alone) and 14 (darunavir-ritonavir plus Echinacea). Mean ritonavir PK parameters were essentially unchanged by coadministration of $E$. purpurea, while small changes in average darunavir PK parameters were noted, with trough concentrations $(\mathrm{C} \tau)$ and measures of total exposure over a complete dosing interval (AUCT) decreasing on average by $16 \%$ and $10 \%$, respectively. However, individual patients displayed declines in darunavir $\mathrm{C} \tau$ or AUC $\tau$ by as much as $40 \%$ and $30 \%$, respectively, while maximum observed darunavir concentrations $\left(\mathrm{C}_{\max }\right)$ were essentially unchanged by E. purpurea coadministration. Overall, the study indicates that high-dose E. purpurea coadministration does not alter the PK of low dose ritonavir and has on average little effect on the exposure characteristics of darunavir. It is to be noted, however, that studies with ritonavir containing anti-HIV combination products provide little assay sensitivity to capture any Echinacea-mediated effects on CYP3A4 activity because ritonavir, as a potent CYP3A4 inhibitor, is likely to mask any less potent influence of Echinacea on CYP3A4 activity. However, the finding that darunavir exposure was reduced in individual patients by 30 to $40 \%$ upon E. purpurea coadministration, would be consistent with the assumption of a modest induction of hepatic CYP3A4 by Echinacea administration as previously reported by Gorski and colleagues [50]. This finding would be further compatible with the hypothesis, that low-dose ritonavir (100 $\mathrm{mg}$ b.i.d.) may not effectively inhibit hepatic CYP3A4 activity in individual patients.

In a comparable study in thirteen healthy subjects $(8 \mathrm{~m} / 5 \mathrm{f})$ receiving a combination of lopinavir/ritonavir $400 / 100 \mathrm{mg}$ b.i.d. for 29.5 days, subjects were treated concomitantly with $E$. purpurea (500 $\mathrm{mg}$ t.i. d.) for 28 days: 14 days in combination with lopinavir/ritonavir and 14 days of E. purpurea alone [102]. Lopinavir and ritonavir plasma concentrations were determined at the end of each treatment period up to $12 \mathrm{~h}$ postdose. In order to assess CYP3A and P-gp activity, subjects also received single oral doses of the probe drugs MDZ $8 \mathrm{mg}$ (CYP3A4) and fexofenadine $120 \mathrm{mg}$ (P-gp/OATP2B1), respectively, before and after the 28 days of E. purpurea. In this study, neither lopinavir nor ritonavir PK were significantly altered by 14 days of E. purpurea coadministration. The post-Echinacea:pre-Echinacea geometric mean ratios (GMRs) for the lopinavir $\mathrm{AUC}_{0-12 \mathrm{~h}}$ and for maximum concentrations $\left(\mathrm{C}_{\max }\right)$ were $0.96(90 \% \mathrm{Cl} 0.83-1.10, \mathrm{p}=0.82)$ and 1.00 (90\% CI 0.88-1.12, p = 0.72), respectively. Conversely, GMRs for the total exposure of $\mathrm{MDZ}\left(\mathrm{AUC}_{0-\infty}\right)$ and oral $\mathrm{MDZ}$ clearance were $0.73(90 \% \mathrm{Cl} 0.61-0.85, \mathrm{p}=0.008)$ and 1.37 (90\% CI 1.10-1.63, $p=0.02$ ), respectively, thereby suggesting the induction of CYP3A4-mediated MDZ clearance. Fexofenadine PK, in contrast, did not significantly differ before and after $E$. purpurea administration $(\mathrm{p}>0.05)$.

Overall, the study outcome regarding the observed changes in CYP3A4 activity is consistent with the results reported by Moltó for the effects of $E$. purpurea supplementation on a darunavir-ritonavir combination [101]. Both studies consistently indicate that E. purpurea coadministration is unlikely to significantly alter the PK of ritonavir-boosted protease inhibitors, most likely due to the presence of the potent CYP3A inhibitor ritonavir, but still may be capable to modestly induce hepatic CYP3A activity. The lack of any effects of $E$. purpurea treatment on the PK of fexofenadine a substrate of several drug transporters including P-gp, OATP1A2, OATP1B1, and OATP2B1 - in the presence of low-dose ritonavir administration is difficult to interpret, because ritonavir is also a potent inhibitor of P-gp and hence, may mask any modest Echinacea-mediated effects on P-gp (ABCB1).

Abdul et al. investigated the PK and PD interactions of Echinacea with warfarin in 12 healthy male subjects who received a single oral $25 \mathrm{mg}$ dose of warfarin alone and after 2 weeks of pretreatment with high-dose Echinacea $(1275 \mathrm{mg}$ four times daily= $5100 \mathrm{mg} /$ day, containing a mixture of $600 \mathrm{mg}$ of E. angustifolia roots and $675 \mathrm{mg}$ of E. purpurea root; standardized to contain $5.75 \mathrm{mg}$ of total alkylamides per tablet) [103]. In this study, the apparent clearance of $(\mathrm{S})$-warfarin was found slightly increased by $9 \%$ (geom. mean ratio $1.09 ; 90 \% \mathrm{CI}$ of ratio $1.01,1.18$ ) during concomitant Echinacea treatment, a negligible change that achieved, however, statistical significance. Accordingly, the total exposure (i.e., $\mathrm{AUC}_{0-\infty}$ ) to (S)-warfarin was increased by $9 \%$ [geom. mean ratio $1.09(1.01,1.18)]$. The exposure characteristics of $(\mathrm{R})$-warfarin were not changed significantly by Echinacea. Similarly, the PD endpoints were not altered significantly by Echinacea with geometric mean ratios of $1.04(0.95,1.13)$ and 1.09 $(0.91,1.31)$ for $I_{N R} \max$ and $A U C_{I N R}$, respectively. As (S)-warfarin is metabolized by CYP2C9 and CYP3A4, and (R)-warfarin is metabolized by CYP3A4 and CYP1A2 [104], it can be concluded that a 2-week treatment with high doses of Echinacea (i.e., $5100 \mathrm{mg} /$ day) does not substantially alter the activities of these CYP enzymes. Overall, the small effect sizes observed are unlikely to be of clinical relevance. 
The effects of Echinacea supplementation on P-gp function was studied in 18 healthy subjects, randomly assigned to receive a standardized Echinacea supplement (267 mg t.i.d. $=801 \mathrm{mg}$ / day) for 14 days [105]. Subjects were also randomized to receive rifampin (300 mg b.i.d., 7 days) and clarithromycin (500 mg b.i.d., 7 days) as positive controls for P-gp induction and inhibition, respectively. Digoxin (Lanoxin ${ }^{\mathrm{TM}} 0.25 \mathrm{mg}$ ), a well-acknowledged P-gp probe, was administered orally before and after each supplementation and control period. Serial digoxin plasma concentrations were obtained over $24 \mathrm{~h}$. Comparisons of area under the curve values $\left(\mathrm{AUC}_{0-3}, \mathrm{AUC}_{0-24}\right)$, terminal disposition half-life $\left(\mathrm{t}_{1 / 2}\right)$, and maximum serum concentrations $\left(C_{\max }\right)$ were used to assess the effects of Echinacea, rifampin, and clarithromycin on digoxin disposition. As expected, rifampin produced significant reductions ( $\mathrm{p}<0.05$ ) in $\mathrm{AUC}_{0-3}, \mathrm{AUC}_{0-24}$, and $\mathrm{C}_{\text {max }}$, while clarithromycin, a known P-gp (ABCB1) inhibitor, increased these parameters significantly $(\mathrm{p}<0.05)$. Echinacea supplementation, in contrast, did not affect digoxin PK, indicating that clinically significant P-gp-mediated herb-drug interactions with Echinacea are unlikely to occur.

In conclusion, the available clinical evidence indicates that Echinacea products at recommended doses do have little potential to cause clinically relevant or worrisome metabolism- and transporter-based PK interactions that involve CYP1A2, CYP2C9, CYP2D6, CYP2E1, and P-gp. In turn, there is consistent clinical evidence suggesting that Echinacea products actually do have potential to modestly induce hepatic CYP3A4 activity, but at the same time also may inhibit the presystemic metabolism of CYP3A4 substrates in the intestine. As both mechanisms in principle counteract each other in terms of the net effects on the systemic exposure of CYP3A4 substrates, may require different times for their onset (i.e., onset of inhibition occurs faster than onset of induction), and also will depend in their effect size on specific substrate characteristics (e.g., oral bioavailability of CYP3A4 substrates), general predictions on the clinical relevance of Echinacea/CYP3A4 substrate interactions are difficult to make. However, caution is advised when CYP3A4 substrates with low oral bioavailability due to pronounced intestinal CYP3A4-mediated metabolism such as verapamil, cyclosporine, or tacrolimus or narrow therapeutic index drugs predominantly cleared by CYP3A4 are coadministered with Echinacea supplementation.

Evidence from two phenotyping studies employing daily Echinacea doses of $1600 \mathrm{mg}[50,100]$ suggests the potential of a weak inhibitory effect of high-dose Echinacea supplementation on CYP1A2 and 2C9. However, reported effect sizes are overall small and therefore unlikely to be of clinical significance in clinical practice, unless substrates with a narrow therapeutic index are concerned, such as warfarin (CYP2C9) or theophylline (CYP1A2). No clinical studies are available addressing the potential impact of Echinacea products on other important metabolic pathways such as CYP2C19 or phase II metabolism (e.g., glucuronidation), and no clinical studies on potential transporter interactions other than P-gp (ABCB1) are available as yet.

\section{Summary and Conclusions of Herb-Drug Interactions in Humans}

$\nabla$

Our review shows that the total number of clinical herb-drug interaction trials with primary PK objectives has increased considerably over the last years. A systematic review of clinical herbdrug interaction studies published in 2005 [106] identified a total of 18 studies only, reporting original drug interaction data on gingko (5 studies), garlic (5), ginseng (3), milk thistle (3), goldenseal and Echinacea (each 1). This comparison indicates that the total number of PK herb-drug interaction studies referring to these six particular herbs has increased by more than 3.5-fold (i.e., from 18 to 66 ) over the last 7 years. Major progress since 2005 has been made for ginkgo (5 vs. 21 studies), milk thistle ( 3 vs. 13 studies), goldenseal ( 1 vs. 8 studies), ginseng ( 3 vs. 9 studies), and Echinacea (1 vs. 7 studies), while only 3 additional studies on garlic were published (5 vs. 8). Emerging contributions of Chinese working groups to this recent development need to be acknowledged in this context. It is also important to note that not just the mere number of studies has increased, but that the overall quality in study design (e.g., employed doses, measures to assess and confirm study sensitivity) and analysis points to a trend towards conceptual improvements.

It is worthy to note that this and virtually all previous reviews on PK-based herb-drug interactions only refer to herbal drugs as perpetrators of PK drug interactions. However, herbal drugs can also be objects of drug interactions (i.e., their exposure can be altered by coadministered perpetrator pharmaceuticals), a mechanism that may be of considerable importance for triggering or aggravating adverse events or secondary PD interactions. It is likely that this particular aspect of herb-drug interactions has not become part of the respective reviews as yet, because of the general paucity of published data. Such studies would require identification of the major active constituents in herbal products along with the establishment of sensitive bioanalytical assays to quantify alterations of the exposure characteristics of herbal drugs due to concomitantly administered perpetrator (i.e., inducer/inhibitors) drugs. As so far significantly underrepresented, the issue of herbal drug products as objects of PK-based drug interactions clearly represents an important field of future work.

Despite the progress that has been made over the last years, the overall number of clinical herb-drug interaction studies appears still limited, at least for some of the herbal products reviewed. Interactions studies with warfarin, a narrow therapeutic index drug and a probe substrate for CYP2C9, are numerous. However, studies with other narrow therapeutic index or critical dose drugs are sparse, except for CsA and antiretroviral drugs. With regards to mechanistic interaction studies, few major CYP enzymes such as CYP1A2, CYP2D6, and CYP3A4 have been well investigated, however the interaction potential of herbal drugs with members of the CYP2C family appears less well established, and we identified only one study addressing a CYP2B family enzyme (CYP2B6) [31]. In this context, however, it is probably also worthy to note that inducibility and susceptibility to inhibition are not of equal clinical relevance for the major CYP enzymes in drug metabolism. CYP2D6 for instance, is hardly inducible at all (i.e., no inducers of CYP2D6 are known), while in turn no potent or moderate in vivo inhibitors of CYP2B6 have been identified as yet [81]. Hence, in vivo studies with herbal products on the inducibility of CYP2D6 and the inhibition of CYP2B6 enzymes are in principle dispensable or at least of secondary importance. In turn, inducibility of CYP1A2, CYP2B6, and CYP3A should always be investigated, when in vitro data indicate induction potential of an herbal product. Systems-pharmacological knowledge on co-regulation of CYP enzymes (e.g,. coinduction of CYP3A and CYP2C enzymes by the pregnane $\mathrm{X}$ receptor) or CYP enzymes and transport proteins (e.g., CYP3A and ABCB1) should also be considered to guide and prioritize smart and efficient in vivo drug interaction studies or programs. 
Studies on the effects of herbal drugs on other important drug metabolizing enzymes, e.g., involved in phase II metabolic reactions (e.g., UGTs, N-acetyltransferases, etc.) are virtually lacking, further highlighting the need for more well-designed clinical trials that will increase our understanding about the underlying mechanisms of herb-drug interactions.

With the exception of ABCB1 and OATP2B1, there is very little information on herb-drug interaction trials investigating the effect on drugs that are mainly absorbed, distributed, or cleared by drug transporters. Given the evidence of clinically relevant drug-drug interactions involving these transporters, there is a still existing need to investigate whether herbal drugs could act as potential perpetrators of these important players in drug absorption, disposition, and elimination.

In light of the limited predictability of herb-drug interactions on the basis of in vitro studies, simultaneous administration of a mixture of substrates of multiple CYP enzymes and transporters in one study (i.e., a "cocktail approach") in human subjects is an attractive approach to evaluate an herbal drug's inhibition or induction potential [107]. There are phenotyping drug cocktails available that are scientifically sound and represent a valuable, safe, and cost-effective tool specifically suited to screen for metabolic and P-gp (ABCB1)-mediated perpetrator interactions of herbal drugs. In case of cocktail or other herb-drug interaction studies relying on the assessment of metabolic ratios, two aspects need to be considered in the assessment of study outcomes: first, urinary metabolic ratios often have a much weaker correlation to enzyme activity and are also not closely correlated to plasmabased metrics. Second, changes in metabolic ratios (regardless of the matrix/compartment concerned, i.e., serum/plasma or urine), i.e., the ratio of its metabolite over its parent compound, are generally greater in magnitude and should not be compared in quantitative terms to changes in standard PK parameters derived from complete plasma concentration-time profiles, such as AUC or clearance. Therefore, it needs to be considered that positive interaction results from studies reporting metabolic ratios always necessitate further in vivo studies to provide quantitative data on exposure changes (AUC, $\mathrm{C}_{\max }$ ) and clearance alterations.

There is an apparent tendency in the published studies to overinterpret and over-communicate the statistical significance of identified herb-drug interactions, without adequate consideration of the overall magnitude of findings and their potential (lack of) clinical significance. One of the possible reasons in that regard might be the frequent reference to metabolic ratios, without adequate consideration how these actually may translate in clearance alterations. The recent FDA Guidance on Drug Interaction Studies lists Echinacea as a weak inhibitor of CYP1A2 and CYP2D6 and gingko as well as goldenseal as weak inhibitors of CYP3A [81]. According to FDA definitions, weak inhibitors are defined as perpetrators increasing the AUC of a sensitive substrate by less than 2 -fold but equal or more than 1.25 -fold or that produce a $20-50 \%$ decrease in clearance. Therefore, the clinical relevance of herb-drug interactions summarized in this review, at first sight, appears to be restricted to interactions with narrow therapeutic index or critical dose drugs. However, it needs also to be acknowledged that categorization of inhibitor/inducer characteristics (i.e., effect sizes) inherently refers to average study outcomes, and that individual responses might differ substantially from population means, based on a variety of extrinsic factors (e.g., diet, nature and number of concomitant medications) and the patients' genetic makeup and metabolizing capacity. Hence, it would be misleading to conclude that herbal drugs conferring only a weak inhibitory/inducing effect on certain transporters/ enzymes would be generally safe in each patient and clinical setting. In this respect, it is interesting to note that the use of herbal drugs appears to be particularly high in patient populations already exposed to complex treatment algorithms and polypharmacotherapy, often involving narrow therapeutic index drugs, e.g., in oncology [108], HIV-infected patients [109], recipients of organ transplants [110], and in the general elderly population, often affected by multimorbidity. Surveys indicate an overall prevalence for herbal preparation use of $13 \%$ to $63 \%$ among cancer patients [111], about two-thirds (67\%) in HIV-infected patients receiving antiretroviral (ARV) therapy [112], and about $75 \%$ and $49 \%$ in dialysis and renal transplant patients, respectively [110]. As in these clinical settings and vulnerable patients even modest herb-drug interaction may contribute to treatment failure (e.g., organ rejection) or increased incidences or severity of adverse events, general caution is advised, and health care professionals in these fields should pay attention to the herbal supplement use of their patients and offer proactive advice in this respect. We do hope that the present review provides adequate and useful scientific support in this regard.

In conclusion, substantial progress has been made over the last years in the clinical assessment of pharmacokinetic herb-drug interactions, as exemplified by the identified clinical study data on six popular herbal drugs. However, there is still a need for additional well-designed clinical trials that will increase our understanding about the underlying mechanisms of herb-drug interactions, and the proper communication of the clinical relevance and implications of respective findings, in order to improve the overall sensitivity of health care professionals and patients and to focus on herb-drug interactions that are likely to be of clinical concern, thereby ultimately enabling and continuously improving informed clinical decision making.

\section{Financial Support}

$\nabla$

No financial support was obtained in the generation of this work.

\section{Conflict of Interest}

$\nabla$

The authors have no conflict of interest to declare.

\section{References}

1 Bent S, Ko R. Commonly used herbal medicines in the United States: a review. Am J Med 2004; 116: 478-485

2 Tindle HA, Davis RB, Phillips RS, Eisenberg DM. Trends in use of complementary and alternative medicine by US adults: 1997-2002. Altern Ther Health Med 2005; 11: 42-49

3 Singh SR, Levine MAH. Natural health product use in Canada: analysis of the National Population Health Survey. Can J Clin Pharmacol 2006; 13: e240-e250

4 Singh SR, Levine MAH. Potential interactions between pharmaceuticals and natural health products in Canada. J Clin Pharmacol 2007; 47: 249-258

5 Kaufman DW, Kelly JP, Rosenberg L, Anderson TE, Mitchell A. Recent patterns of medication use in the ambulatory adult population of the United States: the Slone survey. JAMA 2002; 287: 337-344

6 European medicines Agency (EMA). Guideline on the Investigation of Drug Interactions. CPMP/EWP/560/95/Rev. 1 - Corr.; 22 April 2010. Available at http://www.ema.europa.eu/docs/en_GB/document_ library/Scientific_guideline/2010/05/WC500090112.pdf. Accessed June 06, 2012 
7 Chatterjee P, Franklin MR. Human cytochrome P450 inhibition and metabolic-intermediate complex formation by goldenseal extract and its methylendioxyphenyl components. Drug Metab Dispos 2003; 31: 1391-1397

8 Gurley BJ, Gardner SF, Hubbard MA, Williams DK, Gentry WB, Khan IA, Shah A. In vivo effects of goldenseal, kava kava, black cohosh, and valerian on human cytochrome P450 1A2, 2D6, 2E1, and 3A4/5 phenotypes. Clin Pharmacol Ther 2005; 77: 415-426

9 Gurley BJ, Swain A, Hubbard MA, Hartsfield F, Thaden J, Williams DK, Gentry WB, Tony Y. Supplementation with goldenseal (Hydrastis canadensis), but not kava kava (Piper methysticum), inhibits human CYP3A activity in vivo. Clin Pharmacol Ther 2008; 83: 61-69

10 Guo Y, Chen Y, Tan ZR, Klaassen CD, Zhou HH. Repeated administration of berberine inhibits cytochromes P450 in humans. Eur J Clin Pharmacol 2012; 68: 213-217

11 Izzo AA, Ernst E. Interactions between herbal medicines and prescribed drugs: an updated systematic review. Drugs 2009; 69: 1777-1798

12 Johne A, Roots I. Clinical drug interactions with medicinal herbs. Evid Based Integr Med 2005; 2: 207-228

13 Ulbricht C, Chao W, Costa D, Rusie-Seamon E, Weissner W, Woods J. Clinical evidence of herb-drug interactions: a systematic review by the natural standard research collaboration. Curr Drug Metab 2008, 9: $1063-1120$

14 University of Washington. Metabolism \& Transport Drug Interaction Database (DIDB). Available at http://www.druginteractioninfo.org/. Accessed June 06, 2012

15 DeFeudis FV, Drieu K. Ginkgo biloba extract (EGb 761) and CNS functions: basic studies and clinical applications. Curr Drug Targets 2000 1: $25-58$

16 Kressmann S, Müller WE, Blume HH. Pharmaceutical quality of different Ginkgo biloba brands. J Pharm Pharmacol 2002; 54: 661-669

17 Gurley BJ, Gardner SF, Hubbard MA, Williams DK, Gentry WB, Cui Y, Ang $C Y$. Cytochrome $\mathrm{P} 450$ phenotypic ratios for predicting herb-drug interactions in humans. Clin Pharmacol Ther 2002; 72: 276-287

18 Gurley B, Gardner S, Hubbard M. Clinical assessment of botanical supplementation on cytochrome P450 phenotypes in the elderly: St. John's wort, garlic oil, Panax ginseng, and Ginkgo biloba. Drugs Aging 2005; 22: 525-539

19 Markowitz JS, Donovan JL, Lindsay DeVane C, Sipkes L, Chavin KD. Multiple-dose administration of Ginkgo biloba did not affect cytochrome P-450 2D6 or 3A4 activity in normal volunteers. J Clin Psychopharmacol 2003; 23: 576-581

20 Yasui-Furukori N, Furukori H, Kaneda A, Kaneko S, Tateishi T. The effects of Ginkgo biloba extracts on the pharmacokinetics and pharmacodynamics of donepezil. J Clin Pharmacol 2004; 44: 538-542

21 Robertson SM, Davey RT, Voel J, Formentini E, Alfaro RM, Penzak SR. Effect of Ginkgo biloba extract on lopinavir, midazolam and fexofenadine pharmacokinetics in healthy subjects. Curr Med Res Opin 2008; 24: 591-599

22 Niemi M, Kivistö KT, Hofmann U, Schwab M, Eichelbaum M, Fromm MF. Fexofenadine pharmacokinetics are associated with a polymorphism of the SLCO1B1 gene (encoding OATP1B1). Br J Clin Pharmacol 2005; 59: 602-604

23 Yoshioka M, Ohnishi N, Koishi T, Obata Y, Nakagawa M, Matsumoto T, Tagagi, K, Takara K, Ohkuni T, Yokoyama T, Kuroda K. Studies on interactions between functional foods or dietary supplements and medicines. IV. Effects of Ginkgo biloba leaf extract on the pharmacokinetics and pharmacodynamics of nifedipine in healthy volunteers. Biol Pharm Bull 2004; 27: 2006-2009

24 Uchida S, Yamada H, Li XD, Maruyama S, Ohmori Y, Oki T, Watanabe $H$, Umegaki K, Ohashi K, Yamada S. Effects of Ginkgo biloba extract on pharmacokinetics and pharmacodynamics of tolbutamide and midazolam in healthy volunteers. J Clin Pharmacol 2006; 46: 1290-1298

25 Zuo XC, Zhang BK, Jia SJ, Liu SK, Zhou LY, Li J, Zhang J, Dai LL, Chen BM, Yang GP, Yuan H. Effects of Ginkgo biloba extracts on diazepam metabolism: a pharmacokinetic study in healthy Chinese male subjects. Eur J Clin Pharmacol 2010; 66: 503-509

26 Jiang X, Williams KM, Liauw WS, Ammit AJ, Roufogalis BD, Duke CC, Day $R O$, McLachlan AJ. Effect of ginkgo and ginger on the pharmacokinetics and pharmacodynamics of warfarin in healthy subjects. Br J Clin Pharmacol 2005; 59: 425-432

27 Greenblatt DJ, von Moltke LL, Luo Y, Perloff ES, Horan KA, Bruce A, Reynolds RC, Harmatz JS, Avula B, Khan IA, Goldman P. Ginkgo biloba does not alter clearance of flurbiprofen, a cytochrome P450-2C9 substrate. J Clin Pharmacol 2006; 46: 214-221
28 Mohutsky MA, Anderson GD, Miller JW, Elmer GW. Ginkgo biloba: evaluation of CYP2C9 drug interactions in vitro and in vivo. Am J Ther 2006; 13: $24-31$

29 Yin OQP, Tomlinson B, Waye MMY, Chow AHL, Chow MSS. Pharmacogenetics and herb-drug interactions: experience with Ginkgo biloba and omeprazole. Pharmacogenetics 2004; 14: 841-850

30 Lei HP, Wang G, Wang LS, Ou-yang DS, Chen H, Li Q Zhang W, Tan ZR, Fan $L, H e Y J$, Zhou HH. Lack of effect of Ginkgo biloba on voriconazole pharmacokinetics in Chinese volunteers identified as CYP2C19 poor and extensive metabolizers. Ann Pharmacother 2009; 43: 726-731

31 Lei HP, Ji W, Lin J, Chen H, Tan ZR, Hu DL, Liu LJ, Zhou HH. Effects of Ginkgo biloba extract on the pharmacokinetics of bupropion in healthy volunteers. Br J Clin Pharmacol 2009; 68: 201-206

32 Mauro VF, Mauro LS, Kleshinski JF, Khuder SA, Wang Y, Erhardt PW. Impact of Ginkgo biloba on the pharmacokinetics of digoxin. Am J Ther 2003; 10: 247-251

33 Fan L, Tao GY, Wang G, Chen Y, Zhang W, He YJ, Li Q Lei HP, Jiang F, Hu DL, Huang YF, Zhou HH. Effects of Ginkgo biloba extract ingestion on the pharmacokinetics of talinolol in healthy Chinese volunteers. Ann Pharmacother 2009; 43: 944-949

34 Fan L, Mao XQ Tao GY, Wang G, Jiang F, Chen Y, Li Q, Zhang W, Lei HP, Hu DL, Huang YF, Wang D, Zhou HH. Effect of Schisandra chinensis extract and Ginkgo biloba extract on the pharmacokinetics of talinolol in healthy volunteers. Xenobiotica 2009; 39: 249-254

35 Kudolo GB, Wang W, Javors $M$, Blodgett J. The effect of the ingestion of Ginkgo biloba extract (EGb 761) on the pharmacokinetics of metformin in non-diabetic and type 2 diabetic subjects-a double blind placebocontrolled, crossover study. Clin Nutr 2006; 25: 606-616

36 Zhou M, Xia L, Wang J. Metformin transport by a newly cloned protonstimulated organic cation transporter (plasma membrane monoamine transporter) expressed in human intestine. Drug Metab Dispos 2007; 35: 1956-1962

37 Tzvetkov MV, Saadatmand AR, Lötsch, J, Tegeder I, StinglJC, Brockmöller J. The effects of genetic polymorphisms in the organic cation transporters OCT1, OCT2, and OCT3 on the renal clearance of metformin. Clin Pharmacol Ther 2009; 86: 299-306

38 Lu WJ, Huang JD, Lai ML. The effects of ergoloid mesylates and Ginkgo biloba on the pharmacokinetics of ticlopidine. J Clin Pharmacol 2006 46: 628-634

39 Kim BH, Kim KP, Lim KS, Kim JR, Yoon SH, Cho JY, Lee YO, Lee KH, Jang IJ, Shin SG, Yu KS. Influence of Ginkgo biloba extract on the pharmacodynamic effects and pharmacokinetic properties of ticlopidine: an openlabel, randomized, two-period, two-treatment, two-sequence, singledose crossover study in healthy Korean male volunteers. Clin Ther 2010; 32: 380-390

40 Zadoyan G, Rokitta D, Klement S, Dienel A, Hoerr R, Gramatté T, Fuhr U. Effect of Ginkgo biloba special extract EGb $761^{\circledR}$ on human cytochrome P450 activity: a cocktail interaction study in healthy volunteers. Eur J Clin Pharmacol 2012; 68: 553-560

41 Niemi M, Kivistö KT, Hofmann U, Schwab M, Eichelbaum M, Fromm MF Fexofenadine pharmacokinetics are associated with a polymorphism of the SLCO1B1 gene (encoding OATP1B1). Br J Clin Pharmacol 2005; 59: 602-604

42 Abenavoli L, Aviello G, Capasso R, Milic N, Capasso F. Milk thistle for treatment of nonalcoholic fatty liver disease. Hepat Mon 2011; 11: 173

43 Pradhan SC, Girish C. Hepatoprotective herbal drug, silymarin from experimental pharmacology to clinical medicine. Indian J Med Res 2006; 124: 491-504

44 Brantley SJ, Oberlies NH, Kroll DJ, Paine MF. Two flavonolignans from milk thistle (Silybum marianum) inhibit CYP2C9-mediated warfarin metabolism at clinically achievable concentrations. J Pharmacol Exp Ther 2010; 332: 1081-1087

45 Etheridge AS, Black SR, Patel PR, So J, Mathews JM. An in vitro evaluation of cytochrome P450 inhibition and P-glycoprotein interaction with goldenseal, Ginkgo biloba, grape seed, milk thistle, and ginseng extracts and their constituents. Planta Med 2007; 73: 731-741

46 Sridar C, Goosen T, Kent $U$, Williams J. Silybin inactivates cytochromes P450 3A4 and 2C9 and inhibits major hepatic glucuronosyltransferases. Drug Metab Dispos 2004; 32: 587-594

47 Venkataramanan R, Ramachandran V, Komoroski BJ, Zhang S, Schiff PL, Strom SC. Milk thistle, a herbal supplement, decreases the activity of CYP3A4 and uridine diphosphoglucuronosyl transferase in human hepatocyte cultures. Drug Metab Dispos 2000; 28: 1270-1273 
48 Budzinski JW, Trudeau VL, Drouin CE, Panahi M, Arnason JT, Foster BC. Modulation of human cytochrome P450 3A4 (CYP3A4) and P-glycoprotein (P-gp) in Caco-2 cell monolayers by selected commercialsource milk thistle and goldenseal products. Can J Physiol Pharmacol 2007; 85: 966-978

49 Leber HW, Knauff S. Influence of silymarin on drug metabolizing enzymes in rat and man. Arzneimittelforschung 1976; 26: 1603-1605

50 Gurley BJ, Gardner SF, Hubbard MA, Williams DK, Gentry WB, Carrier J, Khan IA, Edwards DJ, Shah A. In vivo assessment of botanical supplementation on human cytochrome P450 phenotypes: Citrus aurantium, Echinacea purpurea, milk thistle, and saw palmetto. Clin Pharmacol Ther 2004; 76: 428-440

51 Gurley B, Hubbard MA, Williams DK, Thaden J, Tong Y, Gentry WB, Breen $P$, Carrier DJ, Cheboyina S. Assessing the clinical significance of botanical supplementation on human cytochrome P450 3A activity: comparison of a milk thistle and black cohosh product to rifampin and clarithromycin. J Clin Pharmacol 2006; 46: 201-213

52 Fuhr U, Beckmann-Knopp S, Jetter A, Lück H, Mengs U. The effect of silymarin on oral nifedipine pharmacokinetics. Planta Med 2007; 73: 1429-1435

53 Gurley BJ, Swain A, Hubbard MA, Williams DK, Barone G, Hartsfield F, Tong Y, Carrier DJ, Cheboyina S, Battu SK. Clinical assessment of CYP2D6-mediated herb-drug interactions in humans: effects of milk thistle, black cohosh, goldenseal, kava kava, St. John's wort, and Echinacea. Mol Nutr Food Res 2008; 52: 755-763

54 DiCenzo R, Shelton M, Jordan K, Koval C, Forrest A, Reichman R, Morse G. Coadministration of milk thistle and indinavir in healthy subjects. Pharmacotherapy 2003; 23: 866-870

55 Piscitelli S, Formentini E. Effect of milk thistle on the pharmacokinetics of indinavir in healthy volunteers. Pharmacotherapy 2002; 22: 551556

56 Rao BN, Srinivas M, Kumar YS, Rao YM. Effect of silymarin on the oral bioavailability of ranitidine in healthy human volunteers. Drug Metab Drug Interact 2007; 22: 175-185

57 van Erp NP, Baker SD, Zhao M, Rudek MA, Guchelaar HJ, Nortier JW, Sparreboom A, Gelderblom $H$. Effect of milk thistle (Silybum marianum) on the pharmacokinetics of irinotecan. Clin Cancer Res 2005; 11: 78007806

58 Rajnarayana K, Reddy MS, Vidyasagar J, Krishna DR. Study on the influence of silymarin pretreatment on metabolism and disposition of metronidazole. Arzneimittelforschung 2004; 54: 109-113

59 Han Y, Guo D, Chen YY, Tan ZR, Zhou HH. Effect of silymarin on the pharmacokinetics of losartan and its active metabolite E-3174 in healthy Chinese volunteers. Eur J Clin Pharmacol 2009; 65: 585-591

60 Gurley BJ, Barone GW, Williams DK, Carrier J, Breen P, Yates CR, Song PF, Hubbard MA, Tong Y, Cheboyina S. Effect of milk thistle (Silybum marianum) and black cohosh (Cimicifuga racemosa) supplementation on digoxin pharmacokinetics in humans. Drug Metab Disp 2006; 34: 69-74

61 Han Y, Guo D, Chen Y, Tan ZR, Zhou HH. Effect of continuous silymarin administration on oral talinolol pharmacokinetics in healthy volunteers. Xenobiotica 2009; 39: 694-699

62 Hoffmeyer S, Burk O, von Richter O, Arnold HP, Brockmöller J, Johne A, Cascorbi I, Gerloff T, Roots I, Eichelbaum M, Brinkmann U. Functional polymorphisms of the human multidrug-resistance gene: multiple sequence variations and correlation of one allele with P-glycoprotein expression and activity in vivo. Proc Natl Acad Sci USA 2000; 97: 34733478

63 Shirasaka Y, Li Y, Shibue Y, Kuraoka E, Spahn-Langguth H, Kato Y, Langguth P, Tamai I. Concentration-dependent effect of naringin on intestinal absorption of beta(1)-adrenoceptor antagonist talinolol mediated by p-glycoprotein and organic anion transporting polypeptide (Oatp). Pharm Res 2009; 26: 560-567

64 Deng JW, Shon JH, Shin HJ, Park SJ, Yeo CW, Zhou HH, Song IS, Shin JG. Effect of silymarin supplement on the pharmacokinetics of rosuvastatin. Pharm Res 2008; 25: 1807-1814

65 Niemi M. Transporter pharmacogenetics and statin toxicity. Clin Pharmacol Ther 2010; 87: 130-133

66 Barnes PM, Bloom B, Nahin RL. Complementary and alternative medicine use among adults and children: United States, 2007. Natl Health Stat Rep 2008; 12: 1-23

67 Helms S. Cancer prevention and therapeutics: Panax ginseng. Alternat Med Rev 2004; 9: 259-274

68 Attele AS, Wu JA, Yuan CS. Ginseng pharmacology: multiple constituents and multiple actions. Biochem Pharmacol 1999; 58: 1685-1693
69 An G, Morris ME. Herbal supplement-based interactions. In: Pang KS, Rodrigues AD, Peter RM, editors. Enzyme- and transporter-based drug-drug interactions. New York: Springer; 2010: 555-584

70 Anderson GD, Rosito G, Mohustsy MA, Elmer GW. Drug interaction potential of soy extract and Panax ginseng. J Clin Pharmacol 2003; 43: 643-648

71 Donovan JL, DeVane CL, Chavin KD, Taylor RM, Markowitz JS. Siberian ginseng (Eleutheroccus senticosus) effects on CYP2D6 and CYP3A4 activity in normal volunteers. Drug Metab Dispos 2003; 31: 519-522

72 Andrade AS, Hendrix C, Parsons TL, Caballero B, Yuan CS, Flexner CW, Dobs AS, Brown TT. Pharmacokinetic and metabolic effects of American ginseng (Panax quinquefolius) in healthy volunteers receiving the HIV protease inhibitor indinavir. BMC Complement Alternat Med 2008; 8: 50

73 Lee LS, Wise SD, Chan C, Parsons TL, Flexner C, Lietman PS. Possible differential induction of phase 2 enzyme and antioxidant pathways by American ginseng, Panax quinquefolius. J Clin Pharmacol 2008; 48: 599-609

74 Jiang X, Blair EYL, McLachlan AJ. Investigation of the effects of herbal medicines on warfarin response in healthy subjects: a population pharmacokinetic-pharmacodynamic modeling approach. J Clin Pharmacol 2006; 46: 1370-1378

75 Jiang X, Williams KM, Liauw WS, Ammit AJ, Roufogalis BD, Duke CC, Day $R O$, McLachlan AJ. Effect of St John's wort and ginseng on the pharmacokinetics and pharmacodynamics of warfarin in healthy subjects. $\mathrm{Br}$ J Clin Pharmacol 2004; 57: 592-599

76 Yuan CS, Wei G, Dey L, Karrison T, Nahlik L, Maleckar S, Kasza K, Ang-Lee $M$, Moss J. Brief communication: American ginseng reduces warfarin's effect in healthy patients. Ann Intern Med 2004; 141: 23-27

77 Weber HA, Zart MK, Hodges AE, Molloy HM, O'Brien BM, Moody LA, Clark $A P$, Harris RK, Overstreet JD, Smith CS. Chemical comparison of goldenseal (Hydrastis canadensis L.) root powder from three commercial suppliers. J Agric Food Chem 2003; 51: 7352-7358

78 Ettefagh KA, Burns JT, Junio HA, Kaatz GW, Cech NB. Goldenseal (Hydrastis canadensis $\mathrm{L}$.) extracts synergistically enhance the antibacterial activity of berberine via efflux pump inhibition. Planta Med 2011; 77: 835-840

79 Sandhu RS, Prescilla RP, Simonelli TM, Edwards DJ. Influence of goldenseal root on the pharmacokinetics of indinavir. J Clin Pharmacol 2003; 43: $1283-1288$

80 Penzak SR, Acosta EP, Turner M, Edwards DJ, Hon YY, Desai HD, Jann MW. Effect of Seville orange juice and grapefruit juice on indinavir pharmacokinetics. J Clin Pharmacol 2002; 42: 1165-1170

81 US Department of Health and Human Services Food and Drug Administration, Center for Drug Evaluation and Research (CDER). Guidance for industry drug interaction studies - study design, data analysis, implications for dosing, and labeling recommendations (February 2012). Available at http://www.fda.gov/downloads/Drugs/GuidanceComplianceRegulatoryInformation/Guidances/UCM292362.pdf. Accessed June 06, 2012

82 Gurley BJ, Swain A, Barone GW, Williams DK, Breen P, Yates CR, Stuart LB, Hubbard MA, Tong Y, Cheboyina S. Effect of goldenseal (Hydrastis canadensis) and kava kava (Piper methysticum) supplementation on digoxin pharmacokinetics in humans. Drug Metab Dispos 2007; 35: 240-245

$83 \mathrm{Wu}$ X, Li Q Xin H, Yu A, Zhong M. Effects of berberine on the blood concentration of cyclosporin A in renal transplanted recipients: clinical and pharmacokinetic study. Eur J Clin Pharmacol 2005; 61: 567-572

84 Xin HW, Wu XC, Li Q Yu AR, Zhong MY, Liu YY. The effects of berberine on the pharmacokinetics of cyclosporin A in healthy volunteers. Methods Find Exp Clin Pharmacol 2006; 28: 25-29

85 Breithaupt-Grögler K, Ling M, Boudoulas H, Belz GG. Protective effect of chronic garlic intake on elastic properties of aorta in the elderly. Circulation 1997; 96: 2649-2655

86 Silagy CA, Neil HA. A meta-analysis of the effect of garlic on blood pressure. J Hypertens 1994; 12: 463-468

87 Joshi BS, Kaul PN. Alternative medicine: herbal drugs and their critical appraisal - Part I. Prog Drug Res 2001; 56: 1-76

88 Greenblatt DJ, Leigh-Pemberton RA, von Moltke LL. Significance of garlic and its constituents in cancer and cardiovascular disease in vitro interactions of water-soluble garlic components with human. J Nutr 2006; 136: 806-809

89 Lam YWF, Ernst E. Botanical products - drug interactions: focus on garlic, ginkgo and ginseng. In: Lam YWF, Huang SM, Hall SD, editors. Herbal supplements - drug interactions, Volume 162. London: Taylor \& Francis, Inc.; 2006: 107-121 
90 Fisher CD, Augustine LM, Maher JM, Nelson DM, Slitt AL, Klaassen CD, Lehman-McKeeman LD, Cherrington NJ. Induction of drug-metabolizing enzymes by garlic and allyl sulfide compounds via activation of constitutive androstane receptor and nuclear factor E2-related factor 2. Drug Metab Dispos 2007; 35: 995-1000

91 Fukao T, Hosono T, Misawa S, Seki T, Ariga T. The effects of allyl sulfides on the induction of phase II detoxification enzymes and liver injury by carbon tetrachloride. Food Chem Toxicol 2004; 42: 743-749

92 Markowitz JS, Lindsay DeVane C, Chavin KD, Taylor RM, Ruan Y, Donovan JL. Effects of garlic (Allium sativum L.) supplementation on cytochrome P450 2D6 and 3A4 activity in healthy volunteers. Clin Pharmacol Ther 2003; 74: 170-177

93 Loizou GD, Cocker J. The effects of alcohol and diallyl sulphide on CYP2E1 activity in humans: a phenotyping study using chlorzoxazone. Hum Exp Toxicol 2001; 20: 321-327

94 Gwilt PR, Lear CL, Tempero MA, Birt DD, Grandjean AC, Ruddon RW, Nagel $D L$. The effect of garlic extract on human metabolism of acetaminophen. Cancer Res 1994; 3: 155-160

95 Jacek H, Rentsch KM, Steinert HC, Pauli-Magnus C, Meier PJ, Fattinger K. No effect of garlic extract on saquinavir kinetics and hepatic CYP3A4 function measured by the erythromycin breath test. Clin Pharmacol Ther 2004; 75: P80

96 Gallicano K, Foster B, Choudhri S. Effect of short-term administration of garlic supplements on single-dose ritonavir pharmacokinetics in healthy volunteers. Br J Clin Pharmacol 2003; 55: 199-202

97 Lunn DJ, Aarons L. The pharmacokinetics of saquinavir: a Markov chain Monte Carlo population analysis. J Pharmacokinet Biopharm 1998; 26: 47-74

98 Gisolf EH, van Heeswijk RP, Hoetelmans RW, Danner SA. Decreased exposure to saquinavir in HIV-1-infected patients after long-term antiretroviral therapy including ritonavir and saquinavir. AIDS 2000; 14 : 801-805

99 Blumenthal M. The complete German commission E monographs, therapeutic guide to herbal medicines. Austin: American Botanical Council; 1998

100 Gorski JC, Huang SM, Pinto A, Hamman MA, Hilligoss JK, Zaheer NA, Desai $M$, Miller M, Hall SD. The effect of echinacea (Echinacea purpurea root) on cytochrome P450 activity in vivo. Clin Pharmacol Ther 2004; 75: 89-100

101 Moltó J, Valle M, Miranda C, Cedeno S, Negredo E, Barbanoj MJ, Clotet B. Herb-drug interaction between Echinacea purpurea and darunavir-ri- tonavir in HIV-infected patients. Antimicrob Agents Chemother 2011; 55: $326-330$

102 Penzak SR, Robertson SM, Hunt JD, Chairez C, Malati CY, Alfaro RM, Stevenson JM, Kovacs JA. Echinacea purpurea significantly induces cytochrome P450 3A activity but does not alter lopinavir-ritonavir exposure in healthy subjects. Pharmacotherapy 2010; 30: 797-805

103 Abdul MI, Jiang X, Williams KM, Day RO, Roufogalis BD, Liauw WS, Xu H, Matthias A, Lehmann RP, McLachlan AJ. Pharmacokinetic and pharmacodynamic interactions of echinacea and policosanol with warfarin in healthy subjects. Br J Clin Pharmacol 2010; 69: 508-515

104 Wittkowsky AK. Warfarin and other coumarin derivatives: pharmacokinetics, pharmacodynamics, and drug interactions. Semin Vasc Med 2003; 3: 221-230

105 Gurley B, Swain A, Williams D. Gauging the clinical significance of Pglycoprotein-mediated herb-drug interactions: comparative effects of St. John's wort, Echinacea, clarithromycin, and rifampin on dogoxin pharmacokinetics. Mol Nutr Food Res 2008; 52: 772-779

106 Mills E, Wu P, Johnston BC, Gallicano K, Clarke M, Guyatt G. Natural health product-drug interactions: a systematic review of clinical trials. Ther Drug Monit 2005; 27: 549-557

107 Fuhr U, Jetter A, Kirchheiner J. Appropriate phenotyping procedures for drug metabolizing enzymes and transporters in humans and their simultaneous use in the "cocktail" approach. Clin Pharmacol Ther 2007; 81: 270-283

108 Meijerman I, Beijnen JH, Schellens JHM. Herb-drug interactions in oncology: focus on mechanisms of induction. Oncologist 2006; 11: 742752

109 Lee LS, Andrade AS, Flexner C. Interactions between natural health products and antiretroviral drugs: pharmacokinetic and pharmacodynamic effects. Clin Infect Dis 2006; 43: 1052-1059

110 Nowack R, Andrassy J, Fischereder M, Unger M. Effects of dietary factors on drug transport and metabolism: the impact on dosage guidelines in transplant patients. Clin Pharmacol Therap 2009; 85: 439-443

111 Sparreboom A, Cox MC, Acharya MR, Figg WD. Herbal remedies in the United States: potential adverse interactions with anticancer agents. J Clin Oncol 2004; 22: 2489-2503

112 Gore-Felton C, Vosvick M, Power R, Koopman C, Ashton E, Bachmann $M H$, Israelski $D$, Spiegel $D$. Alternative therapies: a common practice among men and women living with HIV. J Assoc Nurses AIDS Care 2003; 14: $17-27$ 